\title{
Crowdsourcing: A Taxonomy and Systematic Mapping Study
}

\author{
Mahmood Hosseini, Alimohammad Shahri, Keith Phalp, Jacqui Taylor, Raian Ali \\ \{mhosseini, ashahri, kphalp, jtaylor,rali\}@bournemouth.ac.uk \\ Bournemouth University, UK
}

\begin{abstract}
Context:

Crowdsourcing, or tapping into the power of the crowd for problem solving, has gained ever-increasing attraction since it was first introduced. Crowdsourcing has been used in different disciplines, and it is becoming well-accepted in the marketplace as a new business model which utilizes Human Intelligence Tasks (HITs).

Objective:

While both academia and industry have extensively delved into different aspects of crowdsourcing, there seems to be no common understanding of what crowdsourcing really means and what core and optional features it has. Also, we still lack information on the kinds and disciplines of studies conducted on crowdsourcing and how they defined it in the context of their application area. This paper will clarify this ambiguity by analysing the distribution and demographics of research in crowdsourcing and extracting taxonomy of the variability and commonality in the constructs defining the concept in the literature.

Method:

We conduct a systematic mapping study and analyse 113 papers, selected via a formal process, and report and discuss the results. The study is combined by a content analysis process to extract a taxonomy of features describing crowdsourcing.

Results:

We extract and describe the taxonomy of features which characterize crowdsourcing in its four constituents; the crowd, the crowdsourcer, the crowdsourced task and the crowdsourcing platform. In addition, we report on different mappings between these features and the characteristics of the studied papers. We also analyse the distribution of the research using multiple criteria and draw conclusions. For example, our results show a constantly increasing interest in the area, especially in North America and a significant interest from industry. Also, we illustrate that although crowdsourcing is shown to be useful in a variety of disciplines, the research in the field of computer science still seems to be dominant in investigating it.

Conclusions:

This study allows forming a clear picture of the research in crowdsourcing and understanding the different features of crowdsourcing and their popularity, what type of research was conducted, where and how and by whom. The study enables researchers and practitioners to estimate the current status of the research in this new field. Our taxonomy of extracted features provides a reference model which could be used to configure crowdsourcing and also define it precisely and make design decisions on which of its variation to adopt.
\end{abstract}

Keywords: Crowdsourcing, Crowdsourcing Features, Systematic Mapping, Taxonomy

\section{Introduction}

Crowdsourcing is a new way of utilizing the power of the crowd in projects which usually require a large number of people, and/or when the costs of their completion by traditional ways, in-house or by outsourcing, is not cost-effective. The term crowdsourcing was first coined in 2006 by Jeff Howe in a companion blog post to his June 2006 Wired Magazine article to refer to such activities [1]. Howe described crowdsourcing as follows:

"Simply defined, crowdsourcing represents the act of a company or institution taking a function once performed by employees and outsourcing it to an undefined 
(and generally large) network of people in the form of an open call. This can take the form of peer-production (when the job is performed collaboratively), but is also often undertaken by sole individuals. The crucial prerequisite is the use of the open call format and the large network of potential laborers."

Since then, crowdsourcing has been used in different domains, but has gained significant attraction in business and marketing fields, due to its capability to provide cheaper labour [2, 3, 4]. The use of crowdsourcing in computer science has also been the centre of attention in recent years, where users are being increasingly seen as the "crowd" that can collectively contribute relevant knowledge to empower software development teams and help plan the adaptation, evolution and maintenance of software [5, 6, 7, 8]. Other domains have also used crowdsourcing where appropriate. For example, it has been recognized in medicine [9, 10, 11] and environmental sciences [12, 13, 14].

There is a wide range of studies in various fields, which investigate, develop and evaluate crowdsourcing. Despite this variety and popularity, the definitions used for crowdsourcing and the nature of application are quite diverse and wide-ranging to the point where the concept seems to lack common meaning. Depending on its utility and implementation, crowdsourcing has been defined in different ways, suiting the needs of a specific application, i.e., the domain has highly influenced the definition. Such diversity in the perception of crowdsourcing has left scholars speculating, among other questions, what exactly crowdsourcing is, and what features make an outsourcing task a crowdsourcing one.

This paper conducts a systematic mapping study on crowdsourcing research. It also extracts a taxonomy of crowdsourcing which offers a clear picture of its core and optional features and how they relate to each other. We analyse how these features map to a wide range of characteristics of the research. We discuss a range of analyses on the current research on crowdsourcing to understand its current status and how and in which direction it is going. The extracted taxonomy of crowdsourcing will be beneficial for researchers and practitioners to understand the diversity and commonality in the definitions of crowdsourcing. It will also help as a reference model of the possible feature configurations of crowdsourcing in its four pillars (the crowd, the crowdsourcer, the crowdsourced task, and the crowdsourcing platform) and how and where these features are typically used.

The paper is structured as follows. Section 2 describes crowdsourcing and its usage in different do- mains. Section 3 explains the objectives of our study. Section 4 explains our research protocol in detail. Section 5 describes how we have extracted features of crowdsourcing. Section 6 analyses our collected data. Section 7 reflects on our analysis results and draws further insights and discusses the threats to validity of our research. Section 8 discusses our future work and concludes the paper.

\section{Crowdsourcing in Brief}

Crowdsourcing is an emerging paradigm which is based on harnessing the power of crowd in solving problems [1]. Crowdsourcing is a form of outsourcing, although it typically does not require a formal contraction which is found in outsourcing tasks to an external organization specialized in that task to perform. Crowdsourcing is also meant to reach a wider range of people, which may sometimes be required to get a solution correctly and efficiently. In certain cases, the reliance on the crowd allows for better solutions in comparison to approaches which rely on an elite group of experts [15].

Crowdsourcing is being used increasingly in different domains of study. One domain of study that has been attracted by the concept of crowdsourcing is computer science. For instance, crowdsourcing has been used in recommendation systems [16], software development [17], multimedia [18], database design [19], and search engine evaluation [20]. Other domains have also utilized crowdsourcing for specific purposes. For example, crowdsourcing has been used in environmental sciences [12, 14], medicine [9, 21], business and management [3, 22], law and politics [23, 24], and sociology [25, 26].

The list of crowdsourcing applications in today's sciences is exhaustive. For example, crowdsourcing has proven useful and applicable in astronomy, with a workshop which was held in March 2014 for the first time in the history of astronomy. This workshop is called "Citizen Science in Astronomy" (Refer to: http://events.asiaa.sinica.edu.tw/workshop/20140303/).

The utilization of crowdsourcing in so many different fields of study should mean that there is already a common definition for crowdsourcing upon which the studies are based. However, by looking at the literature we have observed that crowdsourcing has been defined in different, sometimes contradicted, ways. The variety in these definitions has meant to serve the purpose of that particular study in which crowdsourcing has been employed, but it has also raised confusion on what crowdsourcing really is and what features it holds. For example, Erickson [27] defines crowdsourcing as follows: 
"By 'crowdsourcing' I mean: Tapping the perceptual, cognitive or enactive abilities of many people to achieve a well-defined result such as solving a problem, classifying a data set, or producing a decision."

This definition shows that the crowd involved in crowdsourcing should have some abilities, and that crowdsourcing involves a large number of people. The crowdsourced task in this definition can belong to the problem solving type. This definition of crowdsourcing says nothing about the crowdsourcing platform, e.g., whether it is online or off-line. On the other hand, Li et al. [28] define crowdsourcing as follows:

"Crowdsourcing has recently emerged as a powerful alternative. It outsources tasks to a distributed group of people (usually called workers) who might be inexperienced on these tasks."

In this definition, crowdsourcing is considered to be distributed and the crowd might be inexperienced in the task. The definition does not elaborate on the types of crowdsourced tasks and the characteristics of the crowdsourcing platform and what facilities it should provide. These two definitions view crowdsourcing from two different perspectives, with little or no features in common. Furthermore, the two definitions contradict each other when one paper sees competence as an essential feature of the crowd, while the other observes it as an optional feature of the crowd. While both definitions can be true and worthwhile for the context they are applied in, researchers and practitioners in crowdsourcing may wonder about crowdsourcing features, their essentiality, and their thoroughness. As we will demonstrate in this paper, even though crowdsourcing has been defined differently in the literature, we still need a taxonomy which puts together its various features found in the various definitions.

\section{Systematic Mapping Study: Aim and Objectives}

A systematic mapping is a process of identifying, categorizing, and analysing existing literatures that are relevant to a certain research topic. The result of a systematic mapping will provide a structured report based upon categorizations of the existing literatures, which is often able to illustrate a visual summary that portrays the mapping relationship between the literatures and the categories [29]. The mapping is obtained to identify the extent of the conducted studies, to address any proposed research questions as well as to generate more focus research questions.

Systematic mapping studies have already been proposed in the field of information and software technology and shown useful tools to give a holistic idea of the research in a certain area. For example, Engström and Runeson [30] performed a systematic mapping study on software product line testing. Fernandez et al. [31] performed another mapping study on usability evaluation methods for the web. We follow a similar rationale and perform a systematic mapping study on crowdsourcing. In addition, and while doing the mapping study, we extract a taxonomy of the features of crowdsourcing as a reference model for researchers and practitioners in the field.

We conducted a systematic mapping study on crowdsourcing across various disciplines and fields of study. A mapping study is a preliminary activity of a Systematic Literature Research (SLR), used when there are more extensive research questions, with the intention of identifying the extent of existing studies on a particular topic [32]. Results of this type of study provide an overview of a topic supported by the existing studies including an indication of the quantity of these studies and identification of the primary studies [33].

We conducted this systematic mapping to identify the following:

- the fields of study of the papers which have proposed a definition for crowdsourcing,

- the types of research conducted in those papers,

- the forms of study those papers have followed,

- the venues those papers have been published in,

- the demographics the researchers of those paper represent, and

- the commonality and the variability in the features of crowdsourcing as defined in those papers.

An additional objective of our study is to extract a taxonomy of the commonly used features of crowdsourcing and analyse their distribution across the other characteristics of research and deduce insights and conclusion from that.

The results will provide analyses of the distribution and profile of existing studies. By doing that, our study helps exploring the current status and the degree of maturity of the field and how it is being applied, when, where and by whom. This will be a useful step in identifying gaps in the literature where additional research has to be conducted, as well as areas which got popularity and emphasis more than others. 


\section{Mapping Study: Research Protocol}

To begin our systematic mapping, a set of systematic and structured protocols need to be established to ensure the validity and the value of the mapping result. In our study, we adopted the protocol suggested by Petersen et al. [29]. The final product of the protocol will serve as a manual for conducting a competent mapping, where it will guide the entire research and draw the research boundaries. The mapping process is described as illustrated in Figure 1 .

For the purpose of this mapping study, we identify the papers in different disciplines which have clearly and explicitly defined crowdsourcing. As a main objective of the mapping, we are interested in knowing which features of the crowdsourcing, related to the crowd, the crowdsourcer, the crowdsourced task and the crowdsourcing platform have been mentioned in these definitions. We would also like to know things like the frequency of the features in various crowdsourcing definitions, the necessity or optionality of these features, and the correlation between these features and other attributes of the research including the time and the type of affiliation as well as the field of study.

In the following subsections, we will describe in details the steps for conducting our mapping study. The steps include the definition of the research questions, scope, and search criteria, and determining the selection criteria.

\subsection{Definition of the Research Questions}

This mapping study focuses on the concept of crowdsourcing and the features that are commonly characterizing it across different disciplines and type of studies. Our preliminary research has identified that this research topic has attracted a good number of researchers and has led to several well-known communities (e.g., workshops in top conferences such as VLDB and WWW). However, the perception of crowdsourcing varies a lot in terms of what crowdsourcing really means and how it can be configured in terms of the visible characteristics. In this paper, we adopt the classification developed in [34], which characterizes a crowdsourcing project based on four main pillars (the crowd, the crowdsourcer, the crowdsourced task and the crowdsourcing platform).

To get a clear depiction on the concept of crowdsourcing and the distribution of research on it, this mapping study will focus in addressing the following research questions:

RQ1) What are the main disciplines in which crowdsourcing has been researched?
RQ2) What types of research are used to conduct the study?

RQ3) What forms of study are adopted to conduct the researches of empirical nature?

RQ4) What communities have conducted the study and what types of publication are used to disseminate the research?

RQ5) What are the research trends in the domain?

RQ6) What features are commonly used in crowdsourcing for characterizing its four constituents? Are these features mandatory or optional? And how do they map to the other analyses related to the previous research questions?

\subsection{Definition of Scope}

To frame the above research questions, we also implemented the PICO (Population, Intervention, Comparison, Outcomes) criteria suggested by Petticrew and Roberts [35]. This will help to outline the preliminary scope of the research questions in terms of the requirements that the researchers are willing to seek, and which are relevant to the evidence in the literature. This will provide the research initial boundaries, and therefore serve as an early focus to guide the course of the research.

- Population. Crowdsourcing is a relatively novel idea which was introduced in 2006 and that year is the natural starting year for the search process. Furthermore, we will not confine ourselves to a certain domain or domains of study, or types of study to allow a holistic picture on crowdsourcing. This choice is within the remit of a mapping study. As a result, the population consists of papers from computer science, business and management, law, and other domains of study in which crowdsourcing has been applied. Likewise, the population consists of empirical researches, preliminary studies, and proposed theories at various stages of maturity.

- Intervention. To provide an appropriate mapping, there are certain approaches that should be implemented. These include searching for literatures within the population, extracting information from collected papers, defining a set of classifications, creating the map, and analysing the results to answer the research questions of Section 4.1

- Comparison. Our systematic mapping study compares various dimensions of crowdsourcing. The dimensions include geographical distributions and time distributions, along with other dimensions. We have also chosen a wide range of dimensions based on the content of the papers, such as domains of study, types of authors' affiliation, forms of study, and types of study. 


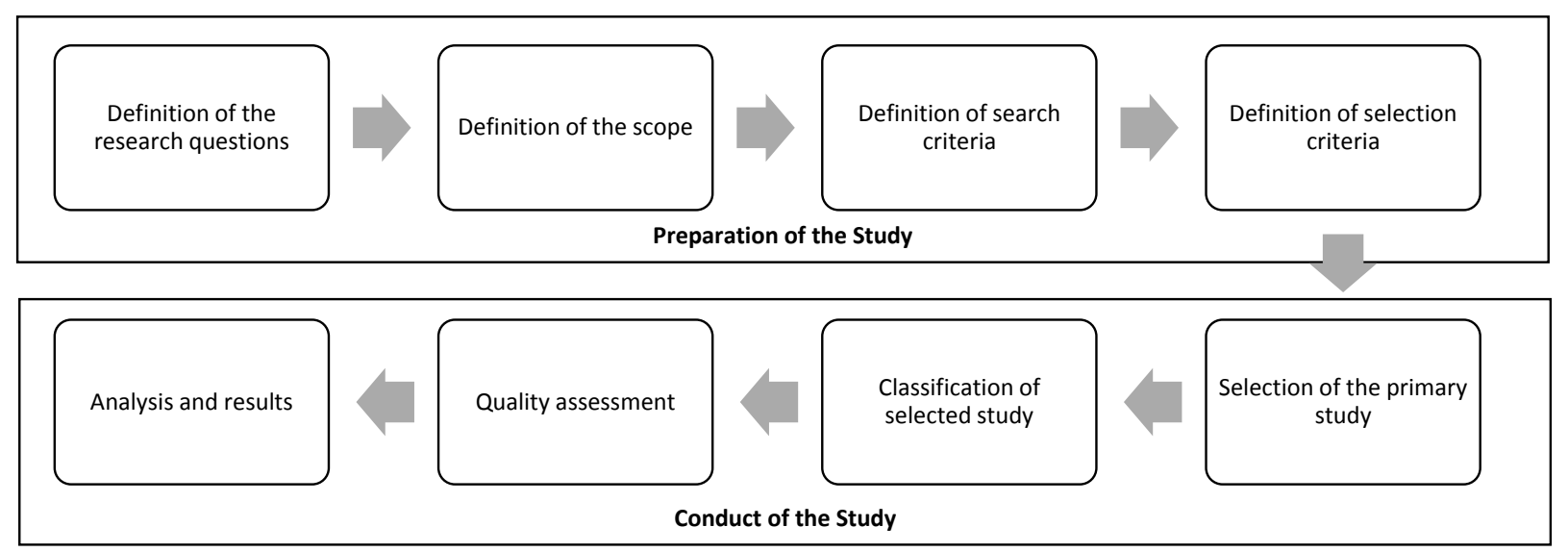

Figure 1: Systematic mapping process

We also cross-tabulate that with the features extracted in the taxonomy of crowdsourcing.

- Outcomes. The collected papers must represent a wide coverage of studied areas in the field of crowdsourcing. This will ensure the validity and the objectivity of this systematic mapping study. We also chose to perform categorization of the existing studies in order to create the map. Finally, an analysis of the map will be done to draw perceptions about the field including the trends and areas which are still under-researched.

\subsection{Definition of Search Criteria}

In conducting our study, we imposed no time criteria on publication year of the papers in order to obtain papers related to crowdsourcing. Since crowdsourcing was introduced in 2006, our time period was naturally from 2006 until January 2014. For each of the papers which we collected, we conducted an initial filtering method to identify the relevance of the paper to this research topics ensuring that the paper was within the scope of this study. We started by reading the titles and the abstracts, and determined the relevant keywords. We selected these keywords based on their relevance to crowdsourcing in the title and abstract. For example, we looked for terms such as crowd, online labour, and Amazon Mechanical Turk. If there were some doubts about the relation between these papers and our study topic, an additional reading through the introduction and the key parts of the paper was required to clarify the relevance. This was to avoid word similarities where the content itself has a different scope than the topic of this research or where the paper was merely an application of crowdsourcing in a certain problem domain without investigating its meaning and definition.

\subsubsection{Keyword Search}

We conducted both automatic and manual searches for papers. We conducted the automatic search by relying on the popular search engines GoogleScholar, DBLP, ScienceDirect, ACM Digital Library, and IEEEXplore. GoogleScholar was particularly helpful because crowdsourcing is a multidisciplinary concept which can be found in a diversity of domains not necessarily indexed by other search engines. This includes studies in business, marketing, management, computing, and medicine. For studies which were found in the search and for which we could not retrieve the manuscript itself, we have tracked the venue in which the study was published and the official website of the authors trying to get it from there. In certain cases, we had to write to the authors directly asking for the manuscript. In this step, we searched for the following search keywords:

- "crowdsource"

- "crowd-source"

- "crowd-sourcing"

- "crowdsourcing"

\subsubsection{Manual Search}

We conducted the manual search in two phases. We conducted the first by tracking researchers who are wellknown for their work in this area, hence finding papers with topics related to this research topic. We selected these well-known authors by searching for them on Google Scholar, by inputting the search string label:crowdsourcing in the search author section. Then 
we went through authors profiles and chose those authors with an established track record on the topic. While it is hard to have concrete metrics of such characteristics, our judgement was informed through looking at the number of publications, the quality of the venue in which these publications were published, the number of citations of the articles and the basic research nature of those publications since we are interested in exploring the concept, not only how it is applied in a specific context. Using this method, we were able to identify 12 well-reputed authors. This also helped us to identify further key researchers, research groups and events by identifying them in the papers which are both cited by, and also citing, the initial set of papers, as we will explain later. The second part of our manual search was to target journals, conferences, symposiums, workshops, and academic technical papers related to crowdsourcing. In this regard, we reviewed papers from the following list of conferences and workshops. Since crowdsourcing is a novel topic, we could not find conferences and workshops related to crowdsourcing which were older than 2009 though individual studies on the concept exist earlier than that date and were found using other forms of our search. The list is as follows:

(a) List of Conferences and Workshops in 2013:

- CHI 2013: Workshop in the ACM SIGCHI Conference on Human Factors in Computing Systems

- CrowdMM 2013: The 2nd International ACM Workshop on Crowdsourcing for Multimedia held in conjunction with ACM Multimedia 2013

- CrowdRec 2013: The first workshop on crowdsourcing and human computation for recommender systems, ACM Conference Series on Recommender Systems (ACM RECSYS)

- CrowdSem 2013: 1st International Workshop on "Crowdsourcing the Semantic Web" in conjunction with the 12th International Semantic Web Conference (ISWC 2013)

- CrowdWork 2013: International Workshop on Crowd Work and Human Computation

- DBCrowd 2013: First VLDB Workshop on Databases and Crowdsourcing

- EC 2013: The 3rd Workshop on Social Computing and User Generated Content

- HCOMP 2013: The First AAAI Conference on Human Computation and Crowdsourcing
- ICWSM 2013 Workshop: Workshop on Social Computing for Workforce 2.0

- Machine Learning Meets Crowdsourcing (WCRWD): ICML'13 Workshop in the 30th International Conference on Machine Learning

- NIPS 2013: Workshop on Crowdsourcing: Theory, Algorithms and Applications

- SoHuman 2: 2nd International Workshop on Social Media for Crowdsourcing and Human Computation

- UbiComp 2013: ACM International Joint Conference on Pervasive and Ubiquitous Computing

(b) List of Conferences and Workshops in 2012:

- AAAI Spring Symposium 2012: Wisdom of the Crowd Workshop

- CHI 2012: Workshop in the ACM SIGCHI Conference on Human Factors in Computing Systems

- Collective Intelligence 2012: Workshop on Collective Intelligence

- CrowdMM 2012: International ACM Workshop on Crowdsourcing for Multimedia held in conjunction with ACM Multimedia 2012

- CrowdSearch 2012: First International Workshop on Crowdsourcing Web Search

- CrowdSens: 1st International Workshop on Multimodal Crowd Sensing

- EC 2012: 2st Workshop on Social Computing and User Generated Content

- HCOMP'12: 4th Workshop on Human Computation

- NIPS 2012: Workshop on Human Computation for Science and Computational Sustainability

- SoHuman2012: 1st International Workshop on Social Media for Human Computation

- Workshop on Machine Learning in Human Computation \& Crowdsourcing: Workshop In Conjunction with ICML 2012

(c) List of Conferences and Workshops in 2011:

- CHI 2011: Workshop on Crowdsourcing and Human Computation 
- EC 2011: 1st Workshop on Social Computing and User Generated Content

- HCOMP'11: 3rd Workshop on Human Computation

- NIPS 2011: Workshop on Computational Social Science and the Wisdom of Crowds

- SIGIR-CIR: 2nd SIGIR Workshop on Crowdsourcing for Information Retrieval

- UbiCrowd'11: 2nd International Workshop on Ubiquitous Crowdsourcing

- WSDM-CSDM 2011: WSDM Workshop on Crowdsourcing for Search and Data Mining (CSDM)

(d) List of Conferences and Workshops in 2010:

- CrowdConf: 1st Annual Conference on the Future of Distributed Work

- CrowdNet: 1st Workshop on Cloud Labor and Human Computation

- CSLDAMT'10: NAACL HLT 2010 Workshop on Creating Speech and Language Data with Amazon's Mechanical Turk.

- CVPRW 2010: IEEE Workshop on Computer Vision and Pattern Recognition

- HCOMP'10: ACM SIGKDD Workshop on Human Computation

- NIPS 2010: Workshop on Computational Social Science and the Wisdom of Crowds

- SIGIR-CSE: 1st SIGIR Workshop on Crowdsourcing for Information Retrieval, CSE 2010

- UbiCrowd'10: 1st International Workshop on Ubiquitous Crowdsourcing

(e) List of Conferences and Workshops in 2009:

- KDD-HCOMP: 1st Human Computation Workshop

\subsubsection{Backward Chaining and Forward Chaining}

To increase the number of collected studies, we also considered a related search derived from the previously identified relevant papers. We realized that most published research provided a background study as a comparison to the conducted study. Therefore, identifying those references was helpful to widen the search boundaries. To do so, we conducted a backward chaining by starting with the papers we obtained from the initial 12 well-reputed authors, looking at the list of references we found in their papers, and referring to those cited papers. We also conducted a forward chaining by referring to the papers which cited the works of these authors. This was mainly performed to ensure that papers which were not included in the keyword search and manual search could also be identified. This helped the identification of anomalous papers within conferences or workshops that were not directly related to this research topic.

\subsection{Definition of Selection Criteria}

Selection criteria define the inclusion and exclusion standards when deciding whether an existing study should be included in the systematic mapping. A paper must meet the conditions defined in the inclusion criteria to be included in the study. The exclusion criteria disqualify papers from those that have already been included.

The inclusion and exclusion standards need to be defined in a clear and objective manner. An explicit set of these standards increase the possibility of producing reliable results and minimizing the possibilities of any harm to the subject. They also define the boundaries that enable other researchers to replicate the search by implementing the same set of standards. However, if the inclusion criteria are too general, they may allow poor quality studies to be included and hence compromise the quality of the final results. On the other hand, if the criteria are too strict, there may be fewer studies included, which might not represent the entire area of study as expected. This is particularly harmful for a mapping study which should cover a discipline in a relatively broad way.

For this study, we defined one clear inclusion criterion. We included any piece of literature (e.g., books, papers, technical reports) that focused on crowdsourcing and provided a clear statement on the meaning of the concept. For example, we took a decision about that by looking at sentences of the type "crowdsourcing means", "crowdsourcing is defined", "crowdsourcing can be viewed", "crowdsourcing refers to" and alike. When there was more than one definition in a paper, we considered the more thorough one.

On the other hand, we defined five exclusion criteria. First, we excluded any study in which crowdsourcing was not defined. Second, we excluded any study where it was defined without mentioning any features of crowdsourcing. In other words, we excluded studies where the definition of the concept was too generic to the point where it does not decide any specifics of the characterizing features. Third, we excluded papers in which the definition was quoted from another paper. In 
this case, we referred to the original paper, if we had not already referred to them, and used that definition. Fourth, we excluded papers which were either unavailable or the availability was only in the form of abstract, tutorials, posters or presentation material. Our reason for this exclusion criterion is that in the absence of the full text we were not able to perform a thorough and trustworthy review. Fifth, not peer-reviewed literatures and duplicated studies were removed from our study.

\subsection{Conduct of the Study}

After we completed our search and a number of literatures were collected, we initiated the main mapping study. We extracted several attributes characterizing the paper such as the year of publication, the venue in which the paper was published, the affiliation country and type of the authors. Then we did the analysis on how the paper defined and perceived crowdsourcing. This was done in two steps. The first step was to find a definition for crowdsourcing by going through the paper in detail, and the second step was to perform a content analysis in the definition in order to extract crowdsourcing features from it.

The first step, definition finding, was performed as follows. We basically read the abstract part, the introduction part and the literature review part to see if we could find any definitions. Here, when we found definitions quoted from other papers, we went to the original paper, double checked the correctness of the quotation and adopted the original definition. However, we then continued reading the original paper to see if it came with its own definition. If no definition were found in the aforementioned parts, we would search the whole paper to see if crowdsourcing was defined in any other part of the paper.

The second step, the feature extraction, was performed after a definition was found. In this step, we performed a content analysis of the definitions to extract features related to the four pillars of crowdsourcing as defined in [34], i.e., the crowd, the crowdsourcer, the crowdsourced task and the crowdsourcing platform. The content analysis was performed based on the definitions we found in the papers under study. At the beginning, the features extraction followed a flat structure. Then, the similar features were grouped into a more abstract feature leading to a hierarchical structure. To avoid confusion, we used a set of controlled vocabulary in order to ensure quality and coherency of the task. For example, words and expressions such as huge, big, large, and vast number were all extracted under the feature titled as "largeness".
While the initial number of papers we found in the area of crowdsourcing was 652 , the results from the exclusion criteria reduced the number of relevant papers to 113 . The classification process was applied to these papers as described in next section.

\subsection{Classification of the Selected Study}

Each of the collected studies was classified into several categories, with the aim of enabling the analysis needed to answer the research questions.

The first classification criterion relates to the areas of study, i.e., the discipline in which crowdsourcing has been defined and used. Though the initial list was large, after going through the selected papers and listing their fields of study and disciplines, the list was much reduced. After deciding the main disciplines where crowdsourcing was used, we also included the "other" option for disciplines that were not listed. We also included the "generic" option, when the study was about crowdsourcing, without any application or reference to any other disciplines. The final list contained the following fields:

\section{- Computer Sciences}

- Business and Management

- Sociology

- Medicine

- Environmental Sciences

- Law

- Other

- Generic

The second classification criterion is about the type of research conducted in the selected papers. According to the classification described by Wieringa et al. [36], types of research of a literature are divided into those of research activities, design activities, or other relevant activities. The following list describes the adaptation types of research:

- Evaluation: Describes an investigation of an existing technique or standards (analyse, assess, and evaluate) in practice to acquire an understanding of a problem

- Validation: Describes an investigation of a novel method or technique that has never been implemented in practice 
- Solution: Proposes new techniques to solve a problem where the technique itself can be either new or a significant extension of an existing technique. It is supported by examples and solid arguments

- Philosophical: Describes the nature of background and knowledge research in presenting a new conceptual framework that implies a new point of view

- Opinion: Describes the author's opinion, values, and preferences without introducing new research results, designs or any conceptual framework

- Experience: Describes the author's personal experience in conducting a practice

The third classification criterion relates to the particular type of empirical studies implemented in the papers which have an empirical nature. Empirical studies are those studies which derive data based upon actual and objective observation or experimentation. There are several forms of empirical study that could be applied to classify a paper such as the one proposed by Bailey et al. [32] and summarized in the following list:

- Case study: Descriptive explanation of empirical inquiries performed in an in-depth study of a particular subject within its real-life context

- Laboratory study: A study using controlled conditions and variables to detect, identify, evaluate, and establish the nature of particular variables in order to investigate an intended result

- Observation: Investigational method conducted systematically to observe behaviour of certain objects without influencing or interfering with the object

- Experiment: Evaluation method to determine if changes applied to certain variables would affect changes in another variable. This study is generally applied to a conditioned environment to ensure its investigation

- Literature review: Informative report upon critical analysis of prior researched studies in a particular subject that describes, summarizes, evaluates, classifies, compares, and clarifies

- Document analysis: A performance of a content systematic examination of documents in order to acquire understanding in a particular subject

- Non-empirical: A description of the researcher's persuasive arguments without data validation obtained from human perception or judgment
In the fourth classification criterion of our mapping process, we also considered the distribution of researchers. This refers to the affiliation in which the researcher or researchers performed the study and whether it is industry or academia. Some studies were performed jointly by both kinds of affiliation and we refer to them as mixed.

The fifth classification criterion adopted in our systematic mapping study is the type of publication. Thus, we categorized the types of publications as journals, conferences, workshops, academic technical reports, and doctoral symposiums. This is important to estimate the stage and maturity of the research.

The sixth classification criterion for our systematic mapping is the publication year. This information will help us to identify the trend of researches in this particular topic.

Finally, we considered the origin of the affiliation which refers to the countries where the affiliations of the authors are located. This is to identify the demographic spread of the interest on research in this particular topic.

\section{Feature Extractions for Crowdsourcing}

As stated earlier, there is a relatively high number of definitions and perceptions of crowdsourcing in the literature. We could find some general observations about the extracted definitions on crowdsourcing:

- The definitions were not notably domain-specific, i.e., they did not seem to be heavily influenced by the field in which the concept was studied and applied. This is an indicator of the uniqueness and the standalone nature of the concept.

- There is no consensus on the set of core features of crowdsourcing. This is an indicator that the concept is still in the exploration stage though it has got its unique nature as discussed in the previous point. For instance, while some studies advocate that incentives should be present to the crowd [12, 37, 38], others do not believe that providing incentives is a fundamental feature of crowdsourcing [2, 39, 40].

- Some of the definitions contradict with each other even for the conditions one should put on crowdsourcing platforms. For example, while some studies considered that crowdsourcing should be performed anonymously [41], others claimed that crowdsourcing requires non-anonymous task completion [12]. 
To clarify and concretely identify this divergence in the understanding of crowdsourcing, we have performed a feature extraction for crowdsourcing using the definition given in the selected papers. We remind here that our selection criteria allowed for including papers which clearly defined and clarified the meaning of crowdsourcing.

A list of collected definitions for crowdsourcing can be found in our academic technical report, available in the link below (http://goo.gl/tCxyT0). For each definition, we investigated the paper directly to extract the definition of crowdsourcing. Then, we extracted the features which were present in the definition.

\section{Analysis and Results}

We have raised a set of research questions in Section 4.1 which highlighted the target of our study and its intended results. Furthermore, we have identified existing studies, analysed the content, and mapped the studies to a set of categories in order to be able to answer these questions. This section presents the finding results and analyses on the categorization from the existing studies, thus addressing each of the previous research questions respectively.

RQ1) What are the main disciplines in which crowdsourcing has been researched?

Figure 2 shows the domains in which crowdsourcing has been defined. Looking at the chart, it can be understood that it is the computer science community which has identified the most definitions for crowdsourcing.

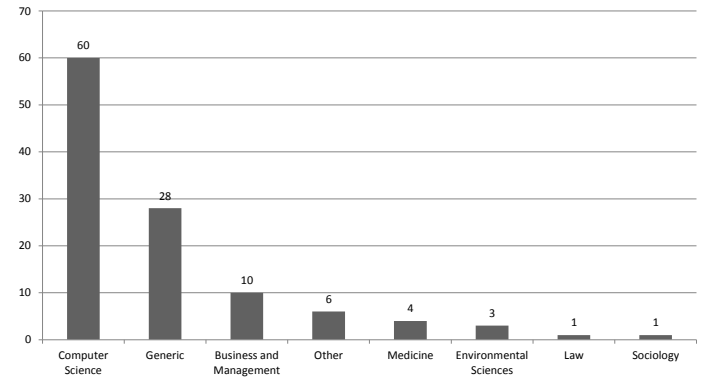

Figure 2: Domains of study of the selected papers

\section{$R Q 2)$ What types of research are used to conduct the} study?

Figure 3 shows the types of study in which crowdsourcing has been defined and investigated. The chart shows that papers with solutions of some kind have mostly tried to define what crowdsourcing is in a clear way. This is perhaps due to the fact that a solution, when validated, requires as a baseline a definition of the feature considered relevant to crowdsourcing.

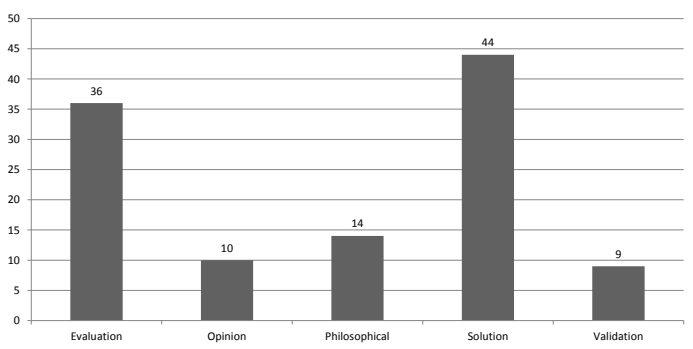

Figure 3: Figure 3: Types of study of the selected papers

RQ3) What forms of study are adopted to conduct the researches of empirical nature?

Figure 4 shows the forms of study in which we could find definitions for crowdsourcing. Most of the papers in which we could find definitions for crowdsourcing were non-empirical papers, with 71 papers out of 113 . Literature review papers and observation papers had the fewest number of definitions, where we could only find one paper.

It should be mentioned that we found papers evaluating a concept or validating it without using any empirical studies, but by means of mathematics. An example of such evaluation papers without an empirical study is the paper by Hirth et al. [42] where the authors use mathematics to analyse two widely used crowd-based approaches to validate the submitted work in Amazon Mechanical Turk and to detect cheating Turkers.

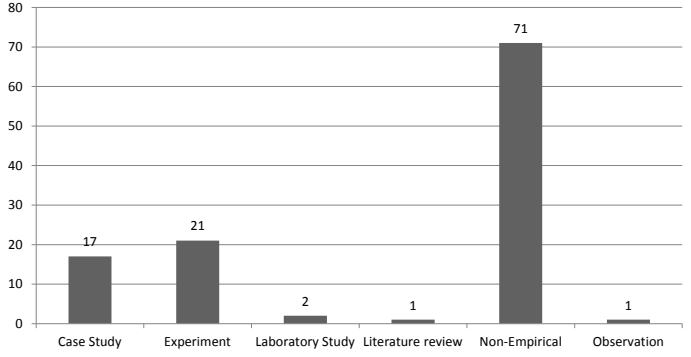

Figure 4: Figure 4: Forms of study of the selected papers

RQ4) What communities have conducted the study and what types of publication are used to disseminate the research?

Figure 5 shows the publication venues where papers with crowdsourcing definitions were found. According to the chart in Figure 5, crowdsourcing was mostly defined in papers found in different workshops. The significant number of papers in conferences and journals 
is an indicator that the concept has started to get consolidated. The high number of papers in workshops is an indicator that research on novel ideas and work in progress is still attractive and the interest in exploring the concept from a diversity of perspectives is still high.

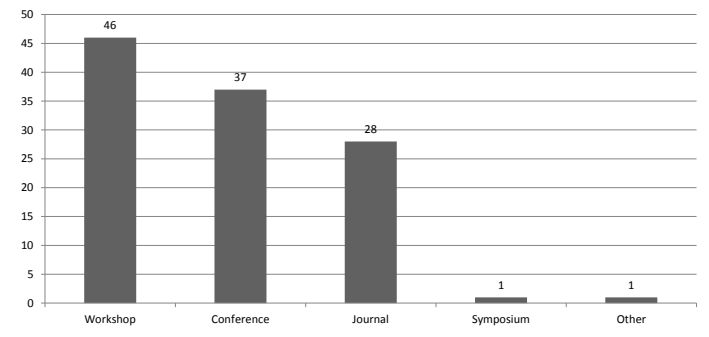

Figure 5: Figure 5: Publication types of the selected papers

$R Q 5)$ What are the research trends in the domain?

Figure 6 shows the affiliation type of our selected papers. Most of the papers come from academia, with 72 papers out of 113 . The reason could be due to the fact that basic or theoretical research is mostly done in academia whereas industry tends to get a working definition or a good practice solution. Still, the accumulation of industry and mixed paper types (41 papers out of 113 papers) shows that the industrial interest in the concept is also significant. We currently have industrial platforms for crowdsourcing such as Amazon Mechanical Turk. This means that the concept was already applied in industry and is being utilized.

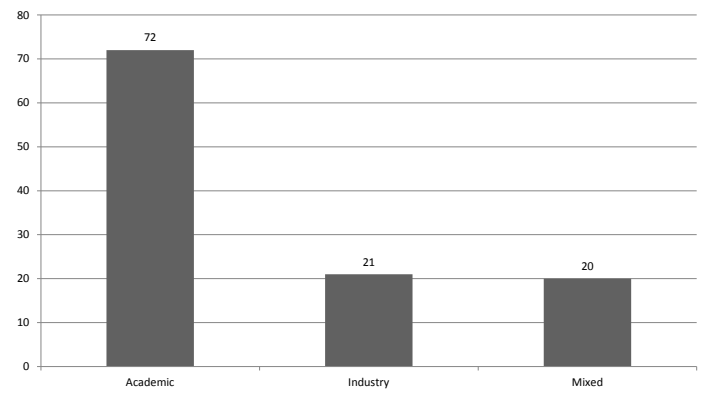

Figure 6: Figure 6: Affiliation for the selected papers

Figure 7 shows the publication year for the studied papers. It is evident that recent years have seen a bigger interest in crowdsourcing and this interest is constant. The list of events listed in Subsection 4.3.2 is another evidence of this stable interest in the concept; the number of events meant for crowdsourcing is also growing.

Figure 8 shows the publication year of the papers classified by the type of affiliation (i.e., academic, industry, or mixed). It can be seen that only in recent years

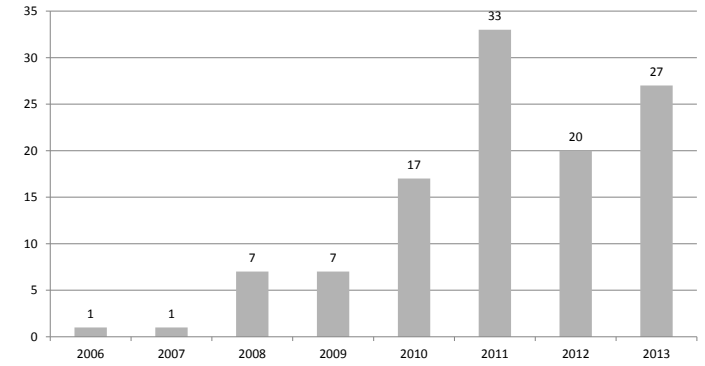

Figure 7: Figure 7: Publication years of the selected papers

(2009 onward) we found papers in mixed affiliations. As for the industry papers, 2011 was the peak year, while for academic papers, there was generally a stable growth. It could be argued that academic papers pay more attention to clearly defining the problem before carrying on with their ideas while industry is mostly based on experience and the product side of the concept. The growth of papers authored by both academia and industry is an indicator that academics and industry experts need the input of each other. For example, in the paper authored by Singer and Mittal [43], first a framework for pricing mechanisms in crowdsourcing platforms is proposed which enables automating the process of pricing and allocation of tasks for requesters in complex markets, and then it is implemented in practice to apply pricing mechanisms in such markets and to give experimental evidence to workers' strategic behaviours in absence of appropriate incentive schemes.

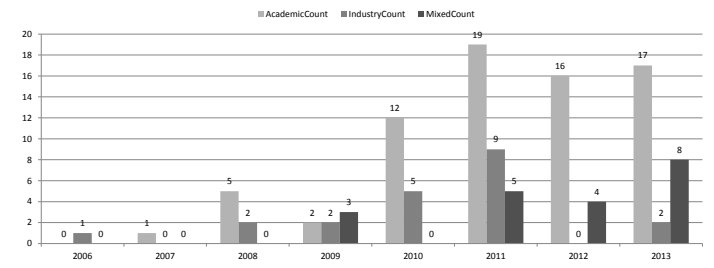

Figure 8: Figure 8: Publication year categorized by affiliation

Figure 9 shows the demographics for the papers studied in this mapping study. Figures 10 and 11 show two more demographic aspects of these papers. According to Figure 9, the United States is by far conducting the most research with 67 papers. Germany and Switzerland follow, by 10 and 9 papers respectively.

Figure 10 shows the categorization of the papers by both their countries and types of affiliation (i.e., academic, industry, or mixed). Again the United States is leading in the academic field by 37 papers, followed by Germany with 8 papers and Switzerland by 6 papers. In 


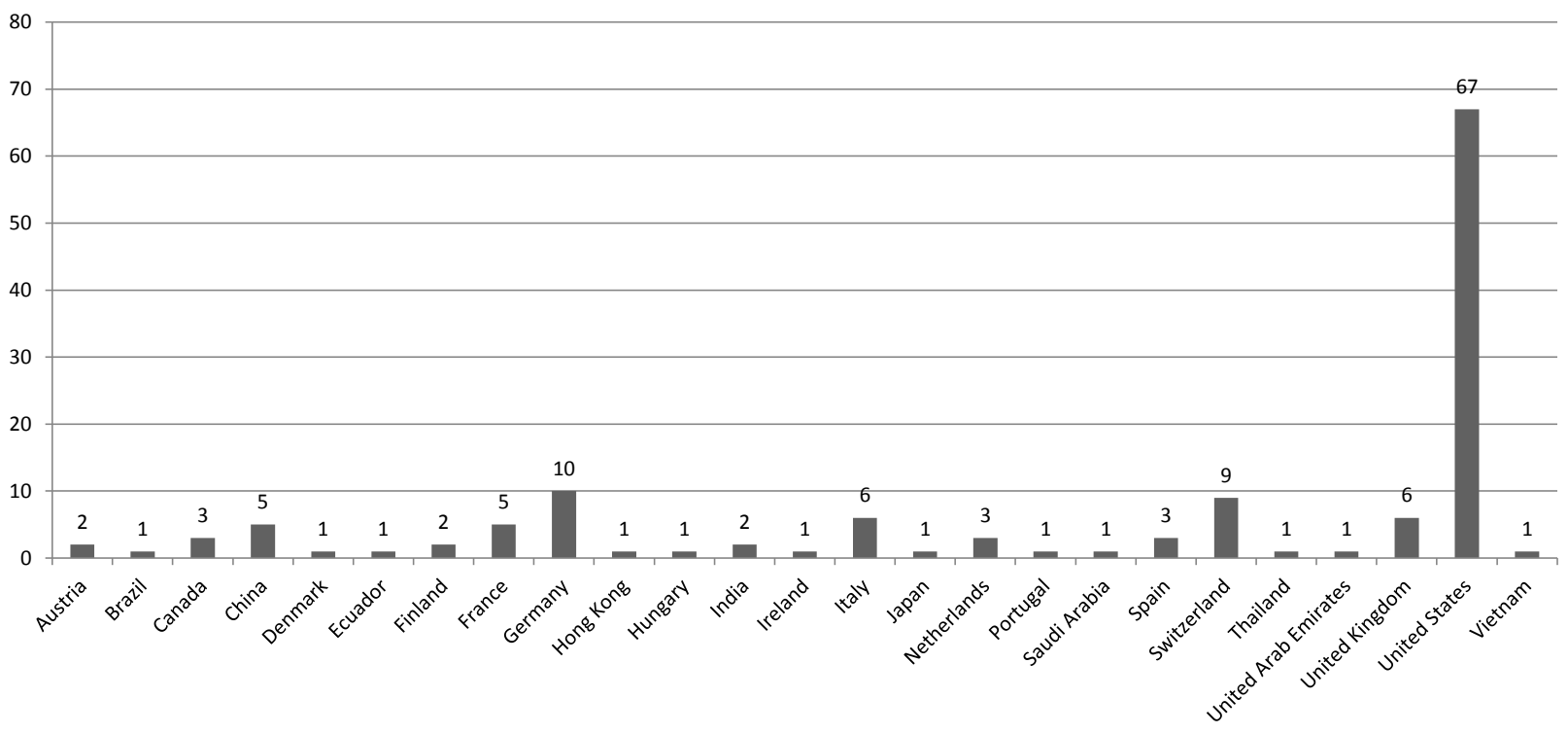

Figure 9: Figure 9: List of countries for the selected papers

the industry, the United States is leading again with 16 papers, followed by India with 2 papers. In the mixed papers, the United States is leading with 14 papers, followed by Switzerland with 3 papers.

Figure 11 shows the same demographics based on the continents in which we found papers with crowdsourcing definitions. Thanks to the United States, North America is leading with 70 papers, followed by Europe with 50 papers. It is interesting to know that no papers from Africa or Oceania (mainly Australia and New Zealand) are present in this domain.

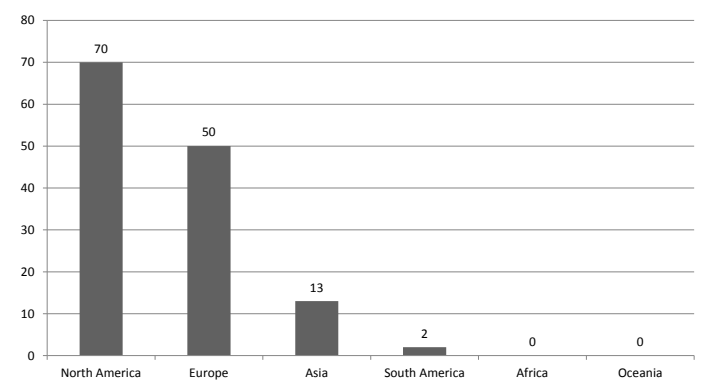

Figure 11: Figure 11: List of continents for the selected papers

The above information could support the planning of research programme at the national and continental level and to estimate the current status of the field and how the research is distributed. Crowdsourcing is heavily reliant on the characteristics of crowd. Thus, it is not straightforward to reuse the results done for a crowd of certain demographics at the global level. As a result, the lack of research in certain geographic areas in this topic puts the application of the achieved results at risk if they are directly reused for the population of other locations. This, together with our statistics, can perhaps encourage research programmes to include crowdsourcing as a priority area as a useful business model.

RQ6) What features are commonly used in crowdsourcing for characterizing its four constituents? Are these features necessity or optional? And how do they map to the other analyses related to the previous research questions?

Before we start the analysis of the features of the four constituents or pillars of crowdsourcing, it is necessary to familiarize ourselves with these features and their meanings in crowdsourcing. Furthermore, it is essential to know how these features can be categorized in order to form a better understanding of the features of these four constituents of crowdsourcing activities.

The first pillar of crowdsourcing is the crowd. After reviewing the current literature on crowdsourcing, we recognized that the crowd of people who participate in a crowdsourcing activity have five distinct features. These features are shown in Table 1, and their definitions are as follows:

- Diversity: diversity is the state or quality of being different or varied. In crowdsourcing, diversity means the recruitment of different people within the crowd to accomplish a task. Such diversity 


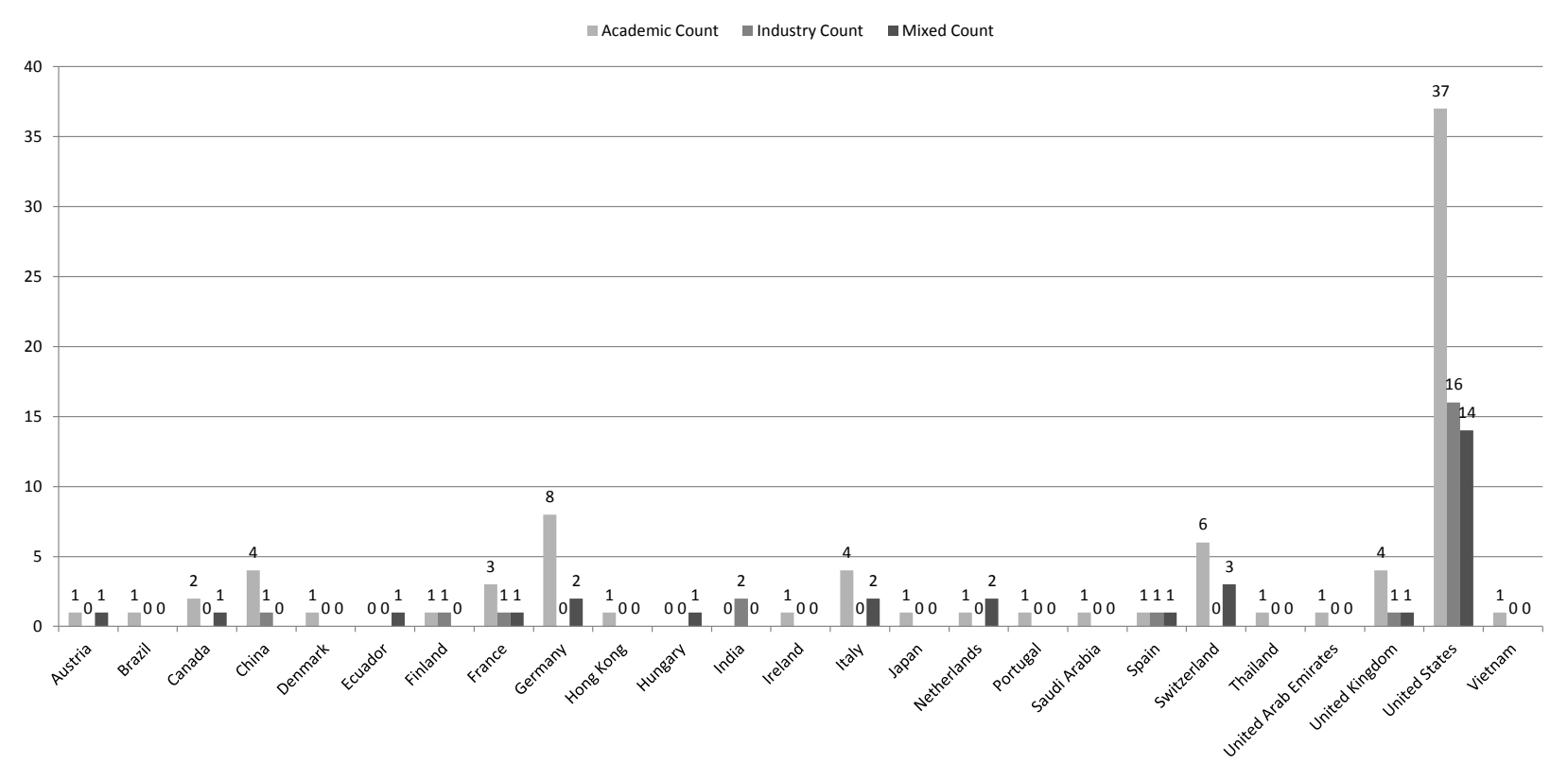

Figure 10: Figure 10: List of countries categorized by affiliation

can be divided into four subcategories. Spatial diversity means recruiting the crowd with different backgrounds and from different locations. Gender diversity means recruiting the crowd with different genders. Age diversity means recruiting the crowd with varied ages. Expertise diversity means recruiting the crowd with diverse experience, expertise and competence.

- Unknown-ness: unknown-ness (or anonymity) is the condition or fact of being anonymous. In crowdsourcing, unknown-ness has one or two of these meanings. The first one is when the crowd participating in a task does not know the crowdsourcer. The second one is when the crowd participating in a task does not know other members of the crowd.

- Largeness: largeness means consisting of big numbers. Largeness also means being comprehensive. In crowdsourcing, largeness occurs when the crowd participating in a crowdsourcing activity is enough to fulfil and achieve the task. However, under certain circumstances, this largeness should not be so abundant to avoid overload, confusion, and unnecessary difficulty in management of the crowd.

- Undefined-ness: undefined-ness means not being determined and not having borders set. In crowd- sourcing, crowd undefined-ness occurs when the crowd is chosen without imposed selection procedures to select a group of people, e.g., people with certain locations, certain abilities, and certain workplaces.

- Suitability: suitability means suiting a given purpose, occasion, condition, etc. In crowdsourcing, crowd suitability means the fit of the crowd to perform a crowdsourcing activity. Such fit could arise when the crowd has certain abilities to perform the task, when the crowd has the ability to collaborate with other members of the crowd, when the crowd volunteers to perform the crowdsourcing activity, and/or when the crowd is motivated in accomplishing a crowdsourcing task. Motivation can be intrinsic or extrinsic. Intrinsic motivations are a feature within the crowd, while extrinsic motivations come from the crowdsourcer, in the form of financial incentives or other forms of incentivizations. Intrinsic motivations are typically the stronger of the two, and can be in the form of mental satisfaction gained from performing a crowdsourcing activity (e.g., the joy of designing a T-shirt cover in Threadless), self-esteem (e.g., knowing the fact that one can design an algorithm for a given problem), personal skill development (e.g., developing personal photography skills through competitions held in iStockPhoto), knowledge sharing through 
crowdsourcing (e.g., Wikipedia), and love of the community in which a crowdsourcing task is being performed (e.g., open-source communities).

Table 1 summarises the features of the crowd, and lists some of the papers from which these features were extracted. In this table and Tables 23 and 4 , we have tried to identify at most four references for every feature and sub-feature of crowdsourcing activity. However, some of these features and sub-features had fewer citing references. For example, we could find self-esteem as a feature of the crowd only in [44]. Furthermore, few of these features and sub-features could not be found explicitly in crowdsourcing definitions in the literature, which means the author(s) did not think of these features as a building block of crowdsourcing. We have illustrated these features and sub-features in the table by $\mathrm{N} / \mathrm{A}$. These features are sometimes counterparts of other features which were explicitly mentioned and hence we deduced them. Moreover, some features are meant for classification only, i.e., concept or aspect features. For example, suitability is an umbrella term for a collection of features and sub-features of the crowd, and was introduced to classify and categorize a number of features and sub-features. We have illustrated this kind of features in the table by $\mathrm{C} / \mathrm{L}$.

The second pillar of crowdsourcing is the crowdsourcer. A crowdsourcer might be an individual, an institution, a non-profit organization, or a company that seeks completion of a task through the power of the crowd. After reviewing the current literature on crowdsourcing, we identified four distinct features of the crowdsourcer. These features are shown in Table 2, and their definitions are as follows:

- Incentives provision: An incentive is something that stimulates one to take action, work harder, etc. It is a kind of stimulus or encouragement. In crowdsourcing, a crowdsourcer may provide incentives as a kind of extrinsic motivation for the crowd. Incentives can be classified in three categories. Probably the most prominent incentive in today's crowdsourcing market is financial incentives. Some crowdsourcing markets, such as Amazon Mechanical Turk, provide payments (sometimes also referred to as micro payments) for the completion of an advertised task. Another type of incentive is social incentives. Some crowds may take part in crowdsourcing activities in order to gain peer recognition [68] or public recognition [20, 44, 66]. The third form of incentives that a crowdsourcer may provide for the crowd is entertainment incentives. Here, the crowdsourcer may
Table 1: List of crowd features

\begin{tabular}{|c|c|}
\hline Crowd Features & Mentioned In \\
\hline 1. Diversity & 12. 44. 45. 46 \\
\hline 1.1. Spatial Diversity & 47. 48. 49. 50 \\
\hline 1.2. Gender Diversity & N/A \\
\hline 1.3. Age Diversity & $\mathrm{N} / \mathrm{A}$ \\
\hline 1.4. Expertise Diversity & {$[2,51,52,53$} \\
\hline 2. Unknown-ness & [54. 55. 56. 57 \\
\hline 2.1. Not Known to Crowdsourcer & [10, 37, 41, 58 \\
\hline 2.2. Not Known to Each Other & [26, 37, 59, 60 \\
\hline 3. Largeness & [2. 59. 61, 62 \\
\hline 3.1. Number Fulfils the Task & $\mathrm{N} / \mathrm{A}$ \\
\hline 3.2. Number Not Abundant & 63 \\
\hline 4. Undefined-ness & [1, 41, 57, 64 \\
\hline 5. Suitability & $\mathrm{C} / \mathrm{L}$ \\
\hline 5.1. Competence & [2, 13, 53, 65 \\
\hline 5.2. Collaboration & [1, 14, 57, 60 \\
\hline 5.3. Volunteering & [21, 44, 45, 51 \\
\hline 5.4. Motivation & [4, 14, 66, 67 \\
\hline 5.4.1. Mental Satisfaction & $\mathrm{N} / \mathrm{A}$ \\
\hline 5.4.2. Self-Esteem & 44 \\
\hline 5.4.3. Personal Skill Development & 44. 68 \\
\hline 5.4.4. Knowledge Sharing & [13. 66 \\
\hline 5.4.5. Love of Community & 69 \\
\hline
\end{tabular}

provide a form of enjoyment or fun in the crowdsourcing activity [20, 66] or may design a game around the crowdsourcing activity [70].

- Open Call: By open call, we mean an audition which is open to anyone who is willing to try out an act. In crowdsourcing, this means that the crowdsourcing activity is mainly open to the general public, and not only to a pre-selected few. An open call provides the opportunity for everyone in the crowd to participate in a crowdsourcing activity. This is synonymous to undefined-ness as a feature of the crowd, but it is from the perspective of the crowdsourcer, i.e., the crowdsourcer may provide an open call so that an undefined group of people participate in a crowdsourcing activity.

- Ethicality provision: An ethical act has to do with ethics or morality. It means conforming to moral standards, or to the standards of conduct of a given profession or group. In crowdsourcing, there are three acts that can be considered ethical during a crowdsourcing activity. The first one is for the 
crowdsourcer to provide an opt-out procedure. The crowdsourcer may provide an opt-out procedure for the crowd, so that the crowd has the right to stop the crowdsourcing activity at any time it wants. Such opting out may or may not affect the participant's condition. For example, the participant may not receive the intended incentive, or they may not be asked to participate in another crowdsourcing activity. Such opting out may or may not affect the crowdsourcer's condition, too. For example, the crowdsourcer may have to provide another open call to obtain the minimum required number of participants in the crowdsourcing activity. Furthermore, this may affect the crowdsourcing time or resources. The second one is for the crowdsourcer to provide feedback to the crowd about the results of the crowdsourced activity, at least upon the crowd's request. This has been observed by some researchers to be an ethical approach [14]. The third one is for the crowdsourcer to ensure that the crowd will not be harmed during the crowdsourcing activity. This is especially important when the crowdsourcing activity is being performed in a real environment, not online.

- Privacy provision: Privacy means the quality or condition of being private. It also means one's private life or personal affair. In crowdsourcing, privacy means that the crowdsourcer should not disclose the crowd's personal and private information to other participants, other organizations and other entities. Privacy provision may also ensure the participating crowd's answers and crowdsourcing activities will not be revealed to others.

Table 2: List of crowdsourcer features

\begin{tabular}{ll}
\hline Crowdsourcer Features & Mentioned In \\
\hline 1. Incentives Provision & {$[44,46,53,69]$} \\
1.1. Financial Incentives & {$[12,63,46,61]$} \\
1.2. Social Incentives & {$[20,44,66,68$} \\
1.3. Entertainment Incentives & {$[20,66,60,62$} \\
\hline
\end{tabular}

\begin{tabular}{ll}
\hline 2. Open Call & {$[1,20,57,58]$} \\
\hline 3. Ethicality Provision & C/L \\
3.1. Opt-out Provision & N/A \\
3.2. Feedback to Crowd & {$[14]$} \\
3.3. No Harm to Crowd & N/A \\
\hline 4. Privacy Provision & N/A \\
\hline
\end{tabular}

The third pillar of crowdsourcing is the crowdsourced task. A crowdsourced task is an outsourced activity that is provided by the crowdsourcer and needs to be completed by the crowd. A crowdsourced task may take different forms. For example, it may be in the form of a problem, an innovation model, a data collection issue, or a fundraising scheme. The crowdsourced task usually needs the expertise, experience, ideas, knowledge, skills, technologies, or money of the crowd. After reviewing the current literature, we identified eight distinct features for the crowdsourced task. These features are shown in Table 3, and their definitions are as follows:

- Traditional operation: By traditional operation, we mean the way the crowdsourced task would be done in an organization if it were not crowdsourced. Without the concept of crowdsourcing, the task would either be done by the employees of the organization, or it would be outsourced to another contractor, agent, company or organization to be completed.

- Outsourcing task: Outsourcing means transferring (certain manufacturing operations, administrative activities, etc.) to outside contractors, esp. so as to reduce one's operating costs. A crowdsourced task is usually a task that would otherwise be outsourced.

- Modularity: Modularity means designating units of standardized size, design, construction, etc. that can be arranged or fitted together in a variety of ways. A crowdsourced task may be an atomic task, but it is usually a more complex task that is broken down into micro tasks to be completed by the crowd.

- Complexity: Complexity is the condition or quality of being complex, not simple or consisting of two or more related parts. A crowdsourced task may be a simple task, or it may be a complex one. The difference between modularity and complexity is that some crowdsourced tasks may be complex, but they may be atomic tasks and not broken down to micro tasks.

- Solvability: Solvability is the capability of a problem to be solved. A crowdsourced task is usually a task that is simple enough for humans to be solved, but too complex for computers. Again, our perspective in this categorization differs from our previous categorizations, as a task may be a complex 
task (complexity: complex) which is not broken down into micro tasks (modularity: atomic) and is nonetheless simple for a human participant to complete (solvability: simple for humans).

- Automation characteristics: Automation, in manufacturing, means a system or method in which many or all of the processes of production, movement, and inspection of parts and materials are automatically performed or controlled by selfoperating machinery, electronic devices, etc. A crowdsourced task is usually a task which is either difficult to automate or expensive to automate (otherwise a computer would solve it instead of crowdsourcing it). The existence of such expense and/or difficulty of task automation opens the way for crowdsourcing. It is worth mentioning that this category focuses on automation, and not complexity or solvability of the task. However, if a task is too complex for computers, it also means that it is too difficult to automate.

- User-driven: A user-driven activity is one that is powered or controlled by users. A crowdsourced task is a user-driven task. Such user-driven activities fall into one of these three subcategories. Sometimes they are in the form of problem solving tasks, which means the crowd should provide a solution to a particular problem. Sometimes they can are innovation tasks, which means the crowd should generate ideas or create designs. Sometimes they are co-creation tasks, which means the crowd participates in a production process in order to create a product.

- Contribution type: When somebody contributes to something, it means that they have a share in bringing about (a result), or are partly responsible for something. A crowdsourced task may be in one of the two contribution forms. First, the contribution of the crowd in the crowdsourced task can be an individual contribution. This means that every participant of the crowd performs the crowdsourced task without helping or getting help from others. Second, the contribution of the crowd in the crowdsourced task can be a collaborative contribution. This means that different participants of the crowd perform the crowdsourced task by acting as a team, pooling resources and working in partnership.

The forth pillar of crowdsourcing is the crowdsourcing platform. The crowdsourcing platform is where the
Table 3: List of crowdsourced task features

\begin{tabular}{|c|c|}
\hline Crowdsourced Task Features & Mentioned In \\
\hline 1. Traditional Operation & [1. $17,64,73$ \\
\hline 1.1. In-house & {$[1,10,54,57]$} \\
\hline 1.2. Outsourced & 54. 57. 74, 75 \\
\hline 2. Outsourcing Task & [1. 61, 64, 73 \\
\hline 3. Modularity & 44 \\
\hline 3.1. Atomic Tasks & N/A \\
\hline 3.2. Divisible to Micro Tasks & {$[16,51,58,69$} \\
\hline 4. Complexity & [44.76 \\
\hline 4.1. Simple Tasks & [13.71.77] \\
\hline 4.2. Complex Tasks & [9. 13, 56, 69) \\
\hline 5. Solvability & $\mathrm{C} / \mathrm{L}$ \\
\hline 5.1. Simple for Humans & [9. 58. 71, 77 \\
\hline 5.2. Complex for Computers & [56. 58, 69. 78 \\
\hline 6. Automation Characteristics & $\mathrm{C} / \mathrm{L}$ \\
\hline 6.1. Difficult to Automate & 48 \\
\hline 6.2. Expensive to Automate & 48 \\
\hline 7. User-driven & [3.79 \\
\hline 7.1. Problem Solving & [20, 27, 63, 69 \\
\hline 7.2. Innovation & [2. 3. 4. 46 \\
\hline 7.3. Co-creation & [3. 65, 68, 74 \\
\hline 8. Contribution Type & $\mathrm{C} / \mathrm{L}$ \\
\hline 8.1. Individual Contribution & {$[1,53,74,80$} \\
\hline 8.2. Collaborative Contribution & {$[1.53$} \\
\hline
\end{tabular}

actual crowdsourcing task takes place. While there are examples of real (off-line or in-person) crowdsourcing platforms [81], the crowdsourcing platform is usually a website, or an online venue. After reviewing the current literature, we identified four distinct features for the crowdsourcing platform. These features are shown in Table 4, and their definitions are as follows:

- Crowd-related interactions: Crowd-related interactions are interactions provided by the crowdsourcing platform between the crowd and the platform. These interactions include, but are not limited to:

- Providing an enrolment mechanism for the crowd to enrol in the crowdsourcing platform.

- Providing an authentication mechanism to authenticate the crowd. 
- Providing a declaration mechanism for the crowd to declare their skills and abilities.

- Providing an assignment mechanism for assigning crowdsourced tasks to the crowd.

- Providing an assistive mechanism to assist the crowd in different activities that happen in the crowdsourcing platform, e.g., helping the crowd to enrol, and helping the crowd to perform the crowdsourced task.

- Providing a submission mechanism for the crowd to submit their results.

- Providing a coordination mechanism to coordinate the crowd during crowdsourcing activities.

- Providing a supervision mechanism to supervise the crowd during crowdsourcing activities.

- Providing a feedback loop mechanism to give feedback to the crowd about their crowdsourcing activities.

- Crowdsourcer-related interactions: Crowdsourcerrelated interactions are interactions provided by the crowdsourcing platform between the crowdsourcer and the platform. These interactions include, but are not limited to:

- Providing an enrolment mechanism for the crowd to enrol in the crowdsourcing platform.

- Providing an authentication mechanism to authenticate the crowd.

- Providing a broadcast mechanism for the crowdsourcer to broadcast their crowdsourced task.

- Providing an assistive mechanism to assist the crowdsourcer in different activities that happen in the crowdsourcing platform, e.g., helping the crowdsourcer to enrol, and helping the crowdsourcer to broadcast the crowdsourced task.

- Providing a time negotiation mechanism for the crowdsourcer to negotiate the deadline or duration of the crowdsourced task with the crowd. This mechanism also allows the crowdsourcer to determine a deadline or a permitted duration without negotiation.
- Providing a price negotiation mechanism for the crowdsourcer to negotiate the financial incentives or rewards of completing the crowdsourced task with the crowd. This mechanism also allows the crowdsourcer to determine a fixed price or a reward without negotiation.

- Providing a verification mechanism for the crowdsourcer to verify the results which are obtained from the crowd.

- Providing a feedback loop mechanism to give feedback to the crowdsourcer about their crowdsourcing activities.

- Task-related facilities: Task-related facilities are facilities provided by the crowdsourcing platform about the crowdsourced task. These facilities include, but are not limited to:

- Providing an aggregation mechanism to aggregate the results of a crowdsourced task. The outcome of such aggregation will be sent to the crowdsourcer for further verification, and may also be partially sent to the crowd as part of the feedback.

- Hiding results obtained from one participant in the crowd from other participants.

- Storing history of completed tasks, either for every task, for every crowdsourcer, for every participant, or a combination of those. Such history may be useful in deciding how future task assignments has to be executed, or in preventing one participant in the crowd from completing one certain crowdsourced task multiple times, etc.

- Providing a threshold mechanism for the quality of the obtained results to ensure a minimum quality is met.

- Providing a threshold mechanism for the quantity of the obtained results to ensure a minimum and/or maximum quantity is met.

- Platform-related facilities: Platform-related facilities are facilities provided by the crowdsourcing platform about the crowdsourcing platform itself. These facilities include, but are not limited to:

- Providing an online environment which is inherent to online crowdsourcing. However, if crowdsourcing is performed in a real (offline or in-person) environment in a particular 
crowdsourcing activity, there should still be an environment in which crowdsourcing will take place.

- Managing platform misuse, either by the crowd or by the crowdsourcer.

- Providing an easy, feasible interface both for the crowd and the crowdsourcer to work.

- Providing an attractive interface both for the crowd and the crowdsourcer to work.

- Providing an interactive interface both for the crowd and the crowdsourcer to work.

- Providing a payment mechanism to allow the crowdsourcer to pay a certain amount of money to the participants. If the reward is something other than money, the platform should also provide mechanisms for it. For example, if the participants should get a certain piece of software or mobile application for free in exchange for their task completion, the platform should provide a download mechanism for the participants.

After a content analysis on crowdsourcing definitions which we could find in the literature, as explained before, we came up with the following list of features and their frequency, as is shown in Tables 5, 6, 7, and 8 for the features of the crowd, the crowdsourcer, the task and the platform respectively. The complete list of definitions and the extracted features from each definition could be found at (http://goo.gl/tCxyT0).

In order to add up the number of times every feature was mentioned in any paper, we acted in the following way. We added the frequency of sub-features with the feature when the feature was not a classification feature. This number is shown in the following four tables as total. We did this because some papers only mentioned a sub-feature in their definition, while from that subfeature the feature itself can be deduced. For instance, when a paper mentions expertise diversity in its definition, it can be deduced that diversity in general is also a feature of crowdsourcing. However, this does not apply for classification features, because these classification features were only created to categorize other features and sub-features, as discussed earlier in this section.

Table 5 shows the features of the crowd and their frequency in crowdsourcing definitions. It can be observed that largeness of the crowd has been mentioned the most, with 59 recurrences in 113 definitions. Diversity of the crowd comes next, with 33 recurrences in 113 definitions. Competence of the crowd is the third
Table 4: List of crowdsourcing platform features

\begin{tabular}{|c|c|}
\hline Crowdsourcing Platform Features & Mentioned In \\
\hline 1. Crowd-related Interactions & $\mathrm{C} / \mathrm{L}$ \\
\hline 1.1. Provide Enrolment & {$[12,65,82,83$} \\
\hline 1.2. Provide Authentication & 65 \\
\hline 1.3. Provide Skill Declaration & [12. 65 \\
\hline 1.4. Provide Task Assignment & $12,45,84,85$ \\
\hline 1.5. Provide Assistance & 63 \\
\hline 1.6. Provide Result Submission & $12,46,53,86$ \\
\hline 1.7. Coordinate Crowd & $60,69,87,88$ \\
\hline 1.8. Supervise Crowd & 84 \\
\hline 1.9. Provide Feedback Loops & 38 \\
\hline 2. Crowdsourcer-related Interactions & $\mathrm{C} / \mathrm{L}$ \\
\hline 2.1. Provide Enrolment & {$[12,65,82,83$} \\
\hline 2.2. Provide Authentication & 65 \\
\hline 2.3. Provide Task Broadcast & [12, 37, 54, 86 \\
\hline 2.4. Provide Assistance & 63 \\
\hline 2.5. Provide Time Negotiation & {$[12$} \\
\hline 2.6. Provide Price Negotiation & {$[12,45$} \\
\hline 2.7. Provide Result Verification & [53, 65 \\
\hline 2.8. Provide Feedback Loops & 38 \\
\hline 3. Task-related Facilities & $\mathrm{C} / \mathrm{L}$ \\
\hline 3.1. Aggregate Results & $62,69,89$ \\
\hline 3.2. Hide Results from Others & [59] \\
\hline 3.3. Store History of Completed Tasks & 65 \\
\hline 3.4. Provide Quality Threshold & [53, 90,91 \\
\hline 3.5. Provide Quantity Threshold & 91 \\
\hline 4. Platform-related Facilities & $\mathrm{C} / \mathrm{L}$ \\
\hline 4.1. Online Environment & 37, 54, 57, 61 \\
\hline 4.2. Manage Platform Misuse & 65 \\
\hline 4.3. Provide Ease of Use & 63 \\
\hline 4.4. Provide Attraction & 63,91 \\
\hline 4.5. Provide Interaction & 59] \\
\hline 4.6. Provide Payment Mechanism & [12, 19,65 \\
\hline
\end{tabular}

most mentioned feature, with 21 recurrences in 113 definitions. Two more prominent features of the crowd in crowdsourcing definitions are motivation of the crowd and unknown-ness of the crowd, with 16 and 14 recurrences respectively. It can be argued that these five features are the most prominent features in crowdsourcing definitions for the crowd who participate in crowdsourcing activities.

Table 6 shows the features of the crowdsourcer and their frequency in crowdsourcing definitions. It can be observed that incentives provision has been mentioned the most, with 61 recurrences in 113 definitions. Financial incentives, which is a sub-feature of incentives provision, has been mentioned 41 times in these definitions, which shows the significance of paid crowdsourc- 
Table 5: List of crowd features and frequencies

\begin{tabular}{ll}
\hline Crowd Features & Frequency \\
\hline 1. Diversity & 21 (total: 33$)$ \\
1.1. Spatial Diversity & 7 \\
1.2. Gender Diversity & 0 \\
1.3. Age Diversity & 0 \\
1.4. Expertise Diversity & 5 \\
\hline 2. Unknown-ness & 5 (total: 14) \\
2.1. Not Known to Crowdsourcer & 5 \\
2.2. Not Known to Each Other & 4 \\
\hline 3. Largeness & 58 (total: 59) \\
3.1. Number Fulfils the Task & 0 \\
3.2. Number Not Abundant & 1 \\
\hline 4. Undefined-ness & 9 (total: 9) \\
\hline 5. Suitability & C/L \\
5.1. Competence & 21 \\
5.2. Collaboration & 10 \\
5.3. Volunteering & 9 \\
5.4. Motivation & 10 (total: 16) \\
5.4.1. Mental Satisfaction & 0 \\
5.4.2. Self-Esteem & 1 \\
5.4.3. Personal Skill Development & 2 \\
5.4.4. Knowledge Sharing & 2 \\
5.4.5. Love of Community & 1 \\
\hline \multicolumn{1}{c}{ Total: 171 recurrences } \\
\hline
\end{tabular}

ing in the literature. Open call comes next, with 29 recurrences in 113 definitions. Despite the expectations, feedback to crowd was mentioned in only one definition, which shows a lack of understanding in how important it really is as an integral part of crowdsourcing.

Table 7 shows the features of the crowdsourced task and their frequency in crowdsourcing definitions. It can be observed that the most prominent feature of a crowdsourced task is that it is user-driven, with 51 recurrences in 113 definitions. Its sub-feature, problem solving, has a high recurrence of 26 in 113 definitions, which shows the prominent way in which crowdsourcing is anticipated to help crowdsourcers. The second most important feature of the crowdsourced task is that it is an outsourcing task, with 27 recurrences in 113 definitions. The third most prominent feature of the crowdsourced task (not considering problem solving as a sub-feature) is its modularity, with 20 recurrences in 113 definitions.

Table 8 shows the features (or facilities) of the crowdsourcing platform and their frequency in crowdsourcing definitions. In 52 definitions out of 113 , this platform is considered to be an online platform. The next two notable features (or facilities) of the crowdsourcing plat-
Table 6: List of crowdsourcer features and frequencies

\begin{tabular}{ll}
\hline Crowdsourcer Features & Frequency \\
\hline 1. Incentives Provision & 10 (total: 61$)$ \\
1.1. Financial Incentives & 41 \\
1.2. Social Incentives & 5 \\
1.3. Entertainment Incentives & 5 \\
\hline 2. Open Call & 29 (total: 29) \\
\hline 3. Ethicality Provision & $\mathrm{C} / \mathrm{L}$ \\
3.1. Opt-out Provision & 0 \\
3.2. Feedback to Crowd & 1 \\
3.3. No Harm to Crowd & 0 \\
\hline 4. Privacy Provision & 0 (total: 0$)$ \\
\hline \multicolumn{2}{c}{ Total: 91 recurrences } \\
\hline
\end{tabular}

form are task broadcasting and result submission facilities with 19 and 6 recurrences respectively. It is also noticeable in comparing this table with the other feature tables that crowdsourcing definitions have scarcely paid attention to the features of crowdsourcing platforms, while their attention has mostly been on features of the crowd who participate in crowdsourcing activities (a total of 171 recurrences for different features of the crowd mentioned in 113 definitions).

Tables 9, 10, 11, and 12 show the distribution of features among academic (ACA), industry (IND), and mixed (MIX) papers. These tables also show the year in which these features emerged (EY). As discusses earlier, this data does not apply to classification type features and thus, we have just put $\mathrm{C} / \mathrm{L}$ in the corresponding fields in these tables. Furthermore, with features that are not present in the literature, as discusses earlier again, we have put N/A in the emerging year field. The numbers in brackets in these tables illustrate the sum of the frequency of a feature and its sub-features, similar to what is present in Tables 5 to 8 .

Table 9 shows the distribution of crowd features among academic, industry, and mixed papers and their emerging year. It can be observed that all different motivation types, i.e., knowledge sharing, love of community, personal skill development, mental satisfaction and self-esteem, are absent in industry papers, and that only knowledge sharing is present in one mixed paper. It can also be observed from Table 9 that not known to crowdsourcer is another absent feature in industry papers, which could probably show the importance of knowing your workers in industry. Another notable absent feature in industry papers is volunteering. This could show either a lack of interest in recruitment type in industry, the focus on the task being done rather than the process 
Table 7: List of crowdsourced task features and frequencies

\begin{tabular}{ll}
\hline Crowdsourced Task Features & Frequency \\
\hline 1. Traditional Operation & 6 (total: 17) \\
1.1. In-house & 6 \\
1.2. Outsourced & 5 \\
\hline 2. Outsourcing Task & 27 (total: 27) \\
\hline 3. Modularity & 1 (total: 20$)$ \\
3.1. Atomic Tasks & 0 \\
3.2. Divisible to Micro Tasks & 19 \\
\hline 4. Complexity & 2 (total: 11) \\
4.1. Simple Tasks & 3 \\
4.2. Complex Tasks & 6 \\
\hline 5. Solvability & C/L \\
5.1. Simple for Humans & 5 \\
5.2. Complex for Computers & 4 \\
\hline 6. Automation Characteristics & C/L \\
6.1. Difficult to Automate & 1 \\
6.2. Expensive to Automate & 1 \\
\hline 7. User-driven & 2 (total: 51$)$ \\
7.1. Problem Solving & 26 \\
7.2. Innovation & 7 \\
7.3. Co-creation & 16 \\
\hline 8. Contribution Type & C/L \\
8.1. Individual Contribution & 5 \\
8.2. Collaborative Contribution & 2 \\
\hline &
\end{tabular}

of task completion, or both.

Table 10 shows the distribution of crowdsourcer features among academic, industry, and mixed papers and their emerging year. The most noticeable concern in this table is the unawareness about ethical issues in crowdsourcing definitions. While there are few examples in the literature that address and discuss ethical issues in crowdsourcing [92], it seems that ethical issues are not fully and duly investigated in crowdsourcing activities. Here, a notable subject is that providing feedback to the crowd, as a sub-feature of ethicality provision, has emerged from mixed papers. This can show the importance of collaboration between academia and industry in inspiring and improving crowdsourcing. The second noticeable thing in this table is that crowdsourcing definitions have also neglected in importance of privacy provision on the crowdsourcer's side. Just like ethicality, there are few examples in the literature addressing privacy [93], but it still needs more investigation into the matter. On the other hand, incentives provision in general, and financial incentives provision in particular
Table 8: List of crowdsourcing platform features (facilities) and frequencies

\begin{tabular}{ll}
\hline Crowdsourcing Platform Features & Frequency \\
\hline 1. Crowd-related Interactions & $\mathrm{C} / \mathrm{L}$ \\
1.1. Provide Enrolment & 5 \\
1.2. Provide Authentication & 1 \\
1.3. Provide Skill Declaration & 2 \\
1.4. Provide Task Assignment & 4 \\
1.5. Provide Assistance & 1 \\
1.6. Provide Result Submission & 6 \\
1.7. Coordinate Crowd & 4 \\
1.8. Supervise Crowd & 1 \\
1.9. Provide Feedback Loops & 1 \\
\hline 2. Crowdsourcer-related Interactions & $\mathrm{C} / \mathrm{L}$ \\
2.1. Provide Enrolment & 5 \\
2.2. Provide Authentication & 1 \\
2.3. Provide Task Broadcast & 19 \\
2.4. Provide Assistance & 1 \\
2.5. Provide Time Negotiation & 1 \\
2.6. Provide Price Negotiation & 2 \\
2.7. Provide Result Verification & 3 \\
2.8. Provide Feedback Loops & 1 \\
\hline 3. Task-related Facilities & $\mathrm{C} / \mathrm{L}$ \\
3.1. Aggregate Results & 3 \\
3.2. Hide Results from Others & 1 \\
3.3. Store History of Completed Tasks & 1 \\
3.4. Provide Quality Threshold & 3 \\
3.5. Provide Quantity Threshold & 1 \\
\hline 4. Platform-related Facilities & $\mathrm{C} / \mathrm{L}$ \\
4.1. Online Environment & 52 \\
4.2. Manage Platform Misuse & 1 \\
4.3. Provide Ease of Use & 1 \\
4.4. Provide Attraction & 2 \\
4.5. Provide Interaction & 1 \\
4.6. Provide Payment Mechanism & 4 \\
\hline & \\
\hline & \\
\hline
\end{tabular}

have been mentioned in many definitions both in academic, industry, and mixed papers. This shows how important it is to provide rewards and incentives to the crowd as an external motivation.

Table 11 shows the distribution of crowdsourced task features among academic, industry, and mixed papers and their emerging year. It is worth noting that industry papers have paid no attention to automation characteristics of crowdsourced tasks. Furthermore, industry papers show no interest in super-feature descriptions such as modularity, complexity and user-driven, while academic papers identified these super-features along with their sub-features. This obviously illustrates the nature of academic and industry papers. On the other hand, the most prominent features of crowdsourced tasks are 
Table 9: List of crowd features and their distribution and emerging year

\begin{tabular}{lllll}
\hline Crowd Features & ACA & IND & MIX & EY \\
\hline 1. Diversity & $11(18)$ & $5(7)$ & $5(8)$ & 2008 \\
1.1. Spatial Diversity & 3 & 2 & 2 & 2010 \\
1.2. Gender Diversity & 0 & 0 & 0 & N/A \\
1.3. Age Diversity & 0 & 0 & 0 & N/A \\
1.4. Expertise Diversity & 4 & 0 & 1 & 2008 \\
\hline 2. Unknown-ness & $4(12)$ & $1(2)$ & $0(0)$ & 2011 \\
2.1. Not Known to Crowdsourcer & 5 & 0 & 0 & 2010 \\
2.2. Not Known to Each Other & 3 & 1 & 0 & 2008 \\
\hline 3. Largeness & $35(36)$ & $13(13)$ & $10(10)$ & 2006 \\
3.1. Number Fulfils the Task & 0 & 0 & 0 & N/A \\
3.2. Number Not Abundant & 1 & 0 & 0 & 2012 \\
\hline 4. Undefined-ness & $5(5)$ & $3(3)$ & $1(1)$ & 2006 \\
\hline 5. Suitability & C/L & C/L & C/L & C/L \\
5.1. Competence & 12 & 5 & 4 & 2008 \\
5.2. Collaboration & 4 & 4 & 2 & 2006 \\
5.3. Volunteering & 7 & 0 & 2 & 2010 \\
5.4. Motivation & $6(11)$ & $3(3)$ & $1(2)$ & 2008 \\
5.4.1. Mental Satisfaction & 0 & 0 & 0 & N/A \\
5.4.2. Self-Esteem & 1 & 0 & 0 & 2012 \\
5.4.3. Personal Skill Development & 2 & 0 & 0 & 2008 \\
5.4.4. Knowledge Sharing & 1 & 0 & 1 & 2011 \\
5.4.5. Love of Community & 1 & 0 & 0 & 2013 \\
\hline & & & &
\end{tabular}

Table 10: List of crowdsourcer features and their distribution and emerging year

\begin{tabular}{lllll}
\hline Crowdsourcer Features & ACA & IND & MIX & EY \\
\hline 1. Incentives Provision & $6(38)$ & $2(16)$ & $2(7)$ & 2009 \\
1.1. Financial Incentives & 26 & 10 & 5 & 2008 \\
1.2. Social Incentives & 3 & 2 & 0 & 2008 \\
1.3. Entertainment Incentives & 3 & 2 & 0 & 2008 \\
\hline 2. Open Call & $14(14)$ & $10(10)$ & $5(5)$ & 2006 \\
\hline 3. Ethicality Provision & $\mathrm{C} / \mathrm{L}$ & $\mathrm{C} / \mathrm{L}$ & $\mathrm{C} / \mathrm{L}$ & $\mathrm{C} / \mathrm{L}$ \\
3.1. Opt-out Provision & 0 & 0 & 0 & N/A \\
3.2. Feedback to Crowd & 0 & 0 & 1 & 2013 \\
3.3. No Harm to Crowd & 0 & 0 & 0 & N/A \\
\hline 4. Privacy Provision & $0(0)$ & $0(0)$ & $0(0)$ & N/A \\
\hline
\end{tabular}

the same in academic, industry and mixed papers. In all categories, the two features of user-driven and outsourcing task for crowdsourced tasks have the highest recurrences $(33,11,7$ and $16,6,5$ for academic, industry and mixed papers respectively). It can be inferred that the view of academia and industry to crowdsourced tasks are identical, which could also lead to the fact that there is a clear and accurate understanding of what crowdsourced tasks really are both in academia and industry.

Table 12 shows the distribution of crowdsourcing platform features among academic, industry, and mixed papers and their emerging year. It is obvious from the table that unlike other pillars of crowdsourcing, there are many features for crowdsourcing platforms that are not mentioned in academic papers (11 out of 28 features), while some of them are mentioned in industry or
Table 11: List of crowdsourced task features and their distribution and emerging year

\begin{tabular}{lllll}
\hline Crowdsourced Task Features & ACA & IND & MIX & EY \\
\hline 1. Traditional Operation & $3(10)$ & $2(5)$ & $1(2)$ & 2006 \\
1.1. In-house & 3 & 2 & 1 & 2006 \\
1.2. Outsourced & 4 & 1 & 0 & 2007 \\
\hline 2. Outsourcing Task & $16(16)$ & $6(6)$ & $5(5)$ & 2006 \\
\hline 3. Modularity & $1(14)$ & $0(3)$ & $0(3)$ & 2012 \\
3.1. Atomic Tasks & 0 & 0 & 0 & N/A \\
3.2. Divisible to Micro Tasks & 13 & 3 & 3 & 2009 \\
\hline 4. Complexity & $2(7)$ & $0(2)$ & $0(2)$ & 2011 \\
4.1. Simple Tasks & 1 & 1 & 1 & 2010 \\
4.2. Complex Tasks & 4 & 1 & 1 & 2011 \\
\hline 5. Solvability & $\mathrm{C} / \mathrm{L}$ & $\mathrm{C} / \mathrm{L}$ & $\mathrm{C} / \mathrm{L}$ & $\mathrm{C} / \mathrm{L}$ \\
5.1. Simple for Humans & 4 & 1 & 0 & 2010 \\
5.2. Complex for Computers & 3 & 0 & 1 & 2011 \\
\hline 6. Automation Characteristics & $\mathrm{C} / \mathrm{L}$ & $\mathrm{C} / \mathrm{L}$ & $\mathrm{C} / \mathrm{L}$ & $\mathrm{C} / \mathrm{L}$ \\
6.1. Difficult to Automate & 1 & 0 & 0 & 2010 \\
6.2. Expensive to Automate & 1 & 0 & 0 & 2010 \\
\hline 7. User-driven & $2(33)$ & $0(11)$ & $0(7)$ & 2012 \\
7.1. Problem Solving & 16 & 7 & 3 & 2008 \\
7.2. Innovation & 6 & 1 & 0 & 2008 \\
7.3. Co-creation & 9 & 3 & 4 & 2007 \\
\hline 8. Contribution Type & $\mathrm{C} / \mathrm{L}$ & $\mathrm{C} / \mathrm{L}$ & $\mathrm{C} / \mathrm{L}$ & $\mathrm{C} / \mathrm{L}$ \\
8.1. Individual Contribution & 2 & 2 & 1 & 2006 \\
8.2. Collaborative Contribution & 1 & 1 & 0 & 2006 \\
\hline & & & &
\end{tabular}

mixed papers. Features such as manage platform misuse and provide feedback loops are among these. This shows a lack of interest in defining crowdsourcing platform features, possibly because it is more practical than theoretical as it mainly deals with implementation issues. If we add the figures in mixed papers definitions to academic definitions, there will be still 8 features of crowdsourcing platforms missing in academic papers. On the other hand, there are 10 features not mentioned in industry papers (e.g., provide quality threshold and provide ease of use) and when we add the figures in mixed papers definitions to industry definitions, there will be still 7 features of crowdsourcing platforms missing in industry papers. This shows that mixed papers have contributed equally to academic and industry interests in defining features of crowdsourcing platforms.

The emerging years for features in crowdsourcing definitions in all four tables (i.e., Tables 9, 10, 11, and 12) show that crowdsourcing is still improving and new ideas and features are added to crowdsourcing almost every year in all four constituents of crowdsourcing. This means that newer features for crowdsourcing activities may still be discovered in the future as crowdsourcing finds its way into new fields of study and dimensions of application.

Tables 13, 14, 15 and 16 show how crowdsourcing features are observed in crowdsourcing definitions in terms of their necessity or optionality. Whether a feature is optional to crowdsourcing or it is necessary for 
Table 12: List of crowdsourcing platform features and their distribution and emerging year

\begin{tabular}{|c|c|c|c|c|}
\hline Crowdsourcing Platform Features & ACA & IND & MIX & EY \\
\hline 1. Crowd-related Interactions & $\mathrm{C} / \mathrm{L}$ & $\mathrm{C} / \mathrm{L}$ & $\mathrm{C} / \mathrm{L}$ & $\mathrm{C} / \mathrm{L}$ \\
\hline 1.1. Provide Enrolment & 1 & 2 & 2 & 2008 \\
\hline 1.2. Provide Authentication & 0 & 1 & 0 & 2009 \\
\hline 1.3. Provide Skill Declaration & 0 & 1 & 1 & 2009 \\
\hline 1.4. Provide Task Assignment & 2 & 0 & 2 & 2009 \\
\hline 1.5. Provide Assistance & 1 & 0 & 0 & 2012 \\
\hline 1.6. Provide Result Submission & 3 & 1 & 2 & 2010 \\
\hline 1.7. Coordinate Crowd & 3 & 1 & 0 & 2011 \\
\hline 1.8. Supervise Crowd & 1 & 0 & 0 & 2013 \\
\hline 1.9. Provide Feedback Loops & 0 & 1 & 0 & 2011 \\
\hline 2. Crowdsourcer-related Interactions & $\mathrm{C} / \mathrm{L}$ & $\mathrm{C} / \mathrm{L}$ & $\mathrm{C} / \mathrm{L}$ & $\mathrm{C} / \mathrm{L}$ \\
\hline 2.1. Provide Enrolment & 1 & 2 & 2 & 2008 \\
\hline 2.2. Provide Authentication & 0 & 1 & 0 & 2009 \\
\hline 2.3. Provide Task Broadcast & 11 & 4 & 4 & 2009 \\
\hline 2.4. Provide Assistance & 1 & 0 & 0 & 2012 \\
\hline 2.5. Provide Time Negotiation & 0 & 0 & 1 & 2012 \\
\hline 2.6. Provide Price Negotiation & 0 & 0 & 2 & 2012 \\
\hline 2.7. Provide Result Verification & 1 & 1 & 1 & 2009 \\
\hline 2.8. Provide Feedback Loops & 0 & 1 & 0 & 2011 \\
\hline 3. Task-related Facilities & $\mathrm{C} / \mathrm{L}$ & $\mathrm{C} / \mathrm{L}$ & $\mathrm{C} / \mathrm{L}$ & $\mathrm{C} / \mathrm{L}$ \\
\hline 3.1. Aggregate Results & 2 & 1 & 0 & 2013 \\
\hline 3.2. Hide Results from Others & 0 & 1 & 0 & 2008 \\
\hline 3.3. Store History of Completed Tasks & 0 & 1 & 0 & 2009 \\
\hline 3.4. Provide Quality Threshold & 3 & 0 & 0 & 2008 \\
\hline 3.5. Provide Quantity Threshold & 1 & 0 & 0 & 2008 \\
\hline 4. Platform-related Facilities & $\mathrm{C} / \mathrm{L}$ & $\mathrm{C} / \mathrm{L}$ & $\mathrm{C} / \mathrm{L}$ & $\mathrm{C} / \mathrm{L}$ \\
\hline 4.1. Online Environment & 37 & 7 & 8 & 2008 \\
\hline 4.2. Manage Platform Misuse & 0 & 1 & 0 & 2009 \\
\hline 4.3. Provide Ease of Use & 1 & 0 & 0 & 2012 \\
\hline 4.4. Provide Attraction & 2 & 0 & 0 & 2008 \\
\hline 4.5. Provide Interaction & 0 & 1 & 0 & 2008 \\
\hline 4.6. Provide Payment Mechanism & 2 & 1 & 1 & 2009 \\
\hline
\end{tabular}

crowdsourcing, was obtained from the definitions found in the literature after a content analysis. Crowdsourcing features were either absent in a paper, mentioned as an essential feature for any crowdsourcing activity, or mentioned as an optional feature. It is worth mentioning that an essential feature does not necessarily mean that it should be present in crowdsourcing, because there are definitions which do not mention these features. The criteria we adopted to classify a feature as essential is that it is mentioned as an essential feature of crowdsourcing in some definitions and other definitions do not argue about its essentiality or do not give a contradicted view. An optional feature is a feature that is mentioned as an optional feature of crowdsourcing in some definitions and other definitions do not argue about its optionality or do not give a contradicted view. However, there is a third kind of feature that has emerged from contradicting definitions of crowdsourcing in the literature.

This third kind of feature is called a debatable feature in this study. Debatable features are features that are deemed essential in some papers, and optional in some others. For example, while some definitions consider competence to be an essential feature of the crowd
[2, 3, 27], there are other definitions that state competence of the crowd is an optional feature [22, 44, 75]. As it is shown in the following four tables, we found that certain features are considered essential (ES) in certain definitions and optional (OP) in others. These are ticked in debatable column (DE) in each table. For features that were not present in any crowdsourcing definitions, we have put N/A in these tables. Also as the rest of this paper, we have put $\mathrm{C} / \mathrm{L}$ for classification-only features. To be spotted easily in these tables, we have only marked the debatable features in its corresponding column.

Table 13 shows the list of crowd features and how crowdsourcing definitions perceive their essentiality. There are four crowd features which are perceived in some definitions as essential to crowdsourcing, while in some other definitions they are perceived as optional. These features are: not known to crowdsourcer, competence, collaboration, and knowledge sharing. The notion whether crowdsourcers should know their workers or not has led to a conflicting view about not known to crowdsourcer. The notion that crowdsourcing can be applied to very simple tasks, such as counting the number of people in a photograph, has led to a conflicting view about competence. The notion that crowdsourcing activities can be done individually in some occasions and collaboratively in other occasions has led to a conflicting view about collaboration. The notion whether the crowd engaging in crowdsourcing activities are sharing their knowledge with other participants in the crowd or not has led to a conflicting view about knowledge sharing. It is also worth mentioning that most of these conflicting views are under the classification of suitability of the crowd for crowdsourcing activities.

On the other hand, diversity, largeness and undefinedness are deemed necessary in all related definitions, while not known to each other is considered to be an optional feature of the crowd in all related definitions.

Table 14 shows the list of crowdsourcer features and how crowdsourcing definitions perceive their essentiality. There are three crowdsourcer features which are perceived in some definitions as essential to crowdsourcing, while in some other definitions they are perceived as optional. These features are: incentives provision, financial incentives and open call.

The notion that crowdsourcing might be done without extrinsic motivations such as incentives has led to a conflicting view about incentives provision. The same can apply to financial incentives. The notion that for certain crowdsourcing activities the crowd should be chosen, i.e., not everyone in the crowd can participate in it if they do not meet certain requirements, has led to a 
Table 13: List of crowd features and their essentiality or optionality

\begin{tabular}{llll}
\hline Crowd Features & ES & OP & DE \\
\hline 1. Diversity & YES & NO & NO \\
1.1. Spatial Diversity & YES & NO & NO \\
1.2. Gender Diversity & N/A & N/A & N/A \\
1.3. Age Diversity & N/A & N/A & N/A \\
1.4. Expertise Diversity & YES & NO & NO \\
\hline 2. Unknown-ness & YES & NO & NO \\
2.1. Not Known to Crowdsourcer & YES & YES & YES \\
2.2. Not Known to Each Other & NO & YES & NO \\
\hline 3. Largeness & YES & NO & NO \\
3.1. Number Fulfils the Task & N/A & N/A & N/A \\
3.2. Number Not Abundant & YES & NO & NO \\
\hline 4. Undefined-ness & YES & NO & NO \\
\hline 5. Suitability & C/L & C/L & C/L \\
5.1. Competence & YES & YES & YES \\
5.2. Collaboration & YES & YES & YES \\
5.3. Volunteering & YES & NO & NO \\
5.4. Motivation & YES & NO & NO \\
5.4.1. Mental Satisfaction & N/A & N/A & N/A \\
5.4.2. Self-Esteem & NO & YES & NO \\
5.4.3. Personal Skill Development & NO & YES & NO \\
5.4.4. Knowledge Sharing & YES & YES & YES \\
5.4.5. Love of Community & NO & YES & NO \\
\hline & & &
\end{tabular}

conflicting view about open call.

On the other hand, social incentives and entertainment incentives are considered to be optional, which probably points to either their insignificance in comparison with financial incentives in crowdsourcing activities, or to their application being still in early stages of consideration and development (e.g., the use of gamification in crowdsourcing activities as an entertainment incentive).

Table 14: List of crowdsourcer features and their essentiality or optionality

\begin{tabular}{llll}
\hline Crowdsourcer Features & ES & OP & DE \\
\hline 1. Incentives Provision & YES & YES & YES \\
1.1. Financial Incentives & YES & YES & YES \\
1.2. Social Incentives & NO & YES & NO \\
1.3. Entertainment Incentives & NO & YES & NO \\
\hline 2. Open Call & YES & YES & YES \\
\hline 3. Ethicality Provision & C/L & C/L & C/L \\
3.1. Opt-out Provision & N/A & N/A & N/A \\
3.2. Feedback to Crowd & YES & NO & NO \\
3.3. No Harm to Crowd & N/A & N/A & N/A \\
\hline 4. Privacy Provision & N/A & N/A & N/A \\
\hline
\end{tabular}

Table 15 shows the list of crowdsourced task features and how crowdsourcing definitions perceive their essentiality. There are nine crowdsourced task features which are perceived in some definitions as essential to crowdsourcing, while in some other definitions they are perceived as optional. The first two are sub-features of traditional operation, i.e., in-house and outsourced. This is because crowdsourced tasks might be traditionally performed inside an organization by its employees, or outsourced to another person or organization. The next two are sub-features of complexity, i.e., simple tasks and complex tasks. This is because crowdsourced tasks might be simple in certain occasions and complex in other occasions. The next three are sub-features of user-driven, i.e., problem solving, innovation and cocreation. This is because crowdsourced tasks are not limited to one form of user-driven activity and can take different forms. The last two are sub-features of contribution type, i.e., individual contribution and collaborative contribution. This is because crowdsourced tasks may be completed individually or collaboratively.

On the other hand, outsourcing task, as a feature of crowdsourced tasks, is deemed necessary in all definitions. Modularity and its sub-feature (i.e., divisible to micro tasks), solvability and its sub-features (i.e., simple for human and complex for computers), and automation characteristics and its sub-features (i.e., difficult to automate and expensive to automate), are also considered to be essential features of crowdsourced tasks.

Table 16 shows the list of crowdsourcing platform features and how crowdsourcing definitions perceive their essentiality. There is only one crowdsourcing platform feature which is perceived in some definitions as essential to crowdsourcing, while in some other definitions it is perceived as optional, and this feature is online environment. This is because crowdsourcing activity may also be done off-line (in-person or in real environment). All other features of crowdsourcing platform are deemed necessary according to crowdsourcing definitions.

\section{Discussion and Reflection}

We have been descriptive in Section 6 In this section, we will elaborate on the meaning of the numbers and statistics presented in the previous section.

\subsection{Study Domain, Study Type and Study Form}

This subsection of our study is related to Research Questions 1,2 and 3. Our findings showed that while crowdsourcing is a multidisciplinary subject, it was mainly studied within the remit of Computer Science field. Out of 113 papers selected and studied in this systematic mapping study, 60 papers were conducted 
Table 15: List of crowdsourced task features and their essentiality or optionality

\begin{tabular}{llll}
\hline Crowdsourced Task Features & ES & OP & DE \\
\hline 1. Traditional Operation & YES & NO & NO \\
1.1. In-house & YES & YES & YES \\
1.2. Outsourced & YES & YES & YES \\
\hline 2. Outsourcing Task & YES & NO & NO \\
\hline 3. Modularity & YES & NO & NO \\
3.1. Atomic Tasks & N/A & N/A & N/A \\
3.2. Divisible to Micro Tasks & YES & NO & NO \\
\hline 4. Complexity & YES & NO & NO \\
4.1. Simple Tasks & YES & YES & YES \\
4.2. Complex Tasks & YES & YES & YES \\
\hline 5. Solvability & C/L & C/L & C/L \\
5.1. Simple for Humans & YES & NO & NO \\
5.2. Complex for Computers & YES & NO & NO \\
\hline 6. Automation Characteristics & C/L & C/L & C/L \\
6.1. Difficult to Automate & YES & NO & NO \\
6.2. Expensive to Automate & YES & NO & NO \\
\hline 7. User-driven & YES & NO & NO \\
7.1. Problem Solving & YES & YES & YES \\
7.2. Innovation & YES & YES & YES \\
7.3. Co-creation & YES & YES & YES \\
\hline 8. Contribution Type & C/L & C/L & C/L \\
8.1. Individual Contribution & YES & YES & YES \\
8.2. Collaborative Contribution & YES & YES & YES \\
\hline & & &
\end{tabular}

under the general theme of Computer Science (Figure 2). This is above 60 percent of the definitions found in our study. Generic papers, which discussed crowdsourcing and defined it for its own sake, came up with 28 definitions. Not surprisingly, Business and Management papers were also active in giving their perspective to crowdsourcing, with 10 definitions. It can be inferred that Computer Sciences and Business and Management are the areas where crowdsourcing has most applications and attraction. We have found papers on other disciplines which we excluded from the study as they did not provide theoretical foundations and concrete specification of the concept. Mostly, those papers applied the concept in its generic meaning, often as getting information from the public. Following our selection criteria presented in Section 4.4 we excluded these papers.

Considering the study types, solution papers were the first with 44 papers, followed by evaluation papers with 36 papers (Figure 3). Knowing that crowdsourcing was first introduced in 2006, the high number of solution and evaluation papers means that the concept is attractive and directly relevant to real world problems. For a new field, we normally expect a greater amount of visionarylike, philosophical and opinion papers. This was not the case here which is another indicator of the practical and
Table 16: List of crowdsourcing platform features and their essentiality or optionality

\begin{tabular}{llll}
\hline Crowdsourcing Platform Features & ES & OP & DE \\
\hline 1. Crowd-related Interactions & C/L & C/L & C/L \\
1.1. Provide Enrolment & YES & NO & NO \\
1.2. Provide Authentication & YES & NO & NO \\
1.3. Provide Skill Declaration & YES & NO & NO \\
1.4. Provide Task Assignment & YES & NO & NO \\
1.5. Provide Assistance & YES & NO & NO \\
1.6. Provide Result Submission & YES & NO & NO \\
1.7. Coordinate Crowd & YES & NO & NO \\
1.8. Supervise Crowd & YES & NO & NO \\
1.9. Provide Feedback Loops & YES & NO & NO \\
\hline 2. Crowdsourcer-related Interactions & C/L & C/L & C/L \\
2.1. Provide Enrolment & YES & NO & NO \\
2.2. Provide Authentication & YES & NO & NO \\
2.3. Provide Task Broadcast & YES & NO & NO \\
2.4. Provide Assistance & YES & NO & NO \\
2.5. Provide Time Negotiation & YES & NO & NO \\
2.6. Provide Price Negotiation & YES & NO & NO \\
2.7. Provide Result Verification & YES & NO & NO \\
2.8. Provide Feedback Loops & YES & NO & NO \\
\hline 3. Task-related Facilities & C/L & C/L & C/L \\
3.1. Aggregate Results & YES & NO & NO \\
3.2. Hide Results from Others & YES & NO & NO \\
3.3. Store History of Completed Tasks & YES & NO & NO \\
3.4. Provide Quality Threshold & YES & NO & NO \\
3.5. Provide Quantity Threshold & YES & NO & NO \\
\hline 4. Platform-related Facilities & C/L & C/L & C/L \\
4.1. Online Environment & YES & YES & YES \\
4.2. Manage Platform Misuse & YES & NO & NO \\
4.3. Provide Ease of Use & YES & NO & NO \\
4.4. Provide Attraction & YES & NO & NO \\
4.5. Provide Interaction & YES & NO & NO \\
4.6. Provide Payment Mechanism & YES & NO & NO \\
\hline & & & \\
\end{tabular}

attractive nature of the field.

With regard to study forms, non-empirical studies held the first position with 71 papers (almost 63 percent), followed by experiment papers with 21 papers and case study papers with 17 papers (Figure 4). This result shows that in the future more work will be needed on empirical studies related to crowdsourcing.

\subsection{Demographics}

This subsection of our study is related to Research Questions 4 and 5. The level of involvement by industry in developing and utilizing crowdsourcing, with clear definition of the concept, was relatively high for an emerging concept. The number of industrial papers was 21 out of 113 papers, embracing a little lower than 20 percent of the literature (Figure 6). Considering the mixed papers, i.e., those conducted jointly by academia and industry, the percentage could rise to about $35 \%$ of the reviewed literature. This shows that crowdsourcing is very attractive industry-wise. The popularity of the 
commercial platform of Amazon Mechanical Turk, for instance, and other crowd work platforms is an evidence of such attraction.

The approximate constant rise in the number of papers with clear crowdsourcing definitions could mean that the field is flourishing and active (Figures 7 and 8). This could also mean that crowdsourcing will continue to flourish in the future with more applications and usages in different fields of study. For example, a new application for crowdsourcing in the field of astronomy, (as discussed in Section 2), shows that in the future, crowdsourcing will be utilized in new domains and fields of study which were not thought of in the past. This clearly illustrates the vibrancy and timeliness of the topic, and that we may encounter even newer applications for crowdsourcing in other fields of study. As a result, this shows that crowdsourcing should be paid more attention to in certain geographic areas where it is still under-researched (Figures 9, 10, 11).

A similar approximate constant rise could be observed in academic papers (Figure 8), while industry papers showed a decline in defining crowdsourcing. It could be interpreted in this way that industry is probably more concerned with the usage of crowdsourcing, while defining the foundations and the semantic of the concept is still one of the main interests of academia.

An interesting finding is that the United States is in a leading position in the field of crowdsourcing, with 67 papers out of 113 papers that we could find in the literature (Figure 9). This means that more than half of the papers were made by the research conducted in the United States, both in academia and industry. Furthermore, the United States was also by far in a leading position with regards to the number of papers across the different categories of the affiliation where the research was conducted, with 37,16 , and 14 papers in academia, industry, and mixed papers respectively (Figure 10). Based on continental distribution of the papers (Figure 11), North America was leading again, thanks to the United States, with 70 papers, with Europe following not very closely with 50 papers. It was somehow unexpected to see no papers from Australia or New Zealand with crowdsourcing definitions. It is worth pointing out again, however, that these results are based on papers with crowdsourcing definitions in them, and do not cover all the papers in crowdsourcing field. Our reason to do that is to exclude papers which utilized the term in a very loose manner, which basically refer to any intervention of the public or even users in contributing information. We considered that too generic to be included, as we previously described in Section 4.4

\subsection{Crowdsourcing Features}

This subsection of our study is related to Research Question 6. While definitions of crowdsourcing were diverse and highly varied, our mapping study yielded some interesting results.

In our study of crowdsourcing features, some of the features were very prominent in the definitions. Incentives provision was the most prominent one, with an overall of 61 occurrences in 113 definitions (with its sub-feature financial incentives being also a prominent feature with 41 occurrences) (Table 6. Largeness was the next mostly cited feature, occurring 59 times in 113 definitions (Table 5). Online environment, userdriven, diversity, and open call followed the trend, with $52,51,33$, and 29 occurrences in crowdsourcing definitions respectively (again, a sub-feature of user-driven, problem solving, was a prominent sub-feature with 26 occurrences). Following these results deduced from the current research in the area, it could be argued that a crowdsourcing platform should try to consider at least these features (or a subset of these) to be rightfully considered a crowdsourcing platform.

The analysis of the distribution of crowdsourcing features in academia and industry for crowd features, crowdsourcer features, and crowdsourced task features produced interesting results (Tables 9, 10, and 11. While academic papers have covered all features except one (i.e., feedback to crowd) when we ignore crowdsourcing platform features, industry papers have not mentioned 11 other features that are found in academic papers. We have intentionally excluded crowdsourcing platform features from this part of the analysis, because it would make this list unnecessarily exhaustive and also because this pillar of crowdsourcing is still being flourished. These features are, alphabetically, as follows:

- Complex for computers: This feature of crowdsourced tasks seems to be not an issue, or otherwise the centre of interest, for industry papers. It can be argued that such inattention is because industry papers are really practical and therefore they have not addressed this issue in any of their definitions.

- Difficult to automate: Industry papers have not mentioned automation issues, one of them being that crowdsourced tasks are more often than not difficult to automate. The reason could be that as mentioned before, industry papers are pragmatic and difficulties in automation or complexities in the crowdsourced task are trivial from industry point of view. 
- Expensive to automate: Automation difficulties, as discussed above, seem to be trivial from industry perspective.

- Expertise diversity: While industry papers do mention diversity as a feature of the crowd, they do not mention this specific diversity. This could be again a pragmatic view to crowdsourcing, which states that while the crowdsourced task is being done successfully, it is trivial whether the crowd participating in the task are diverse in expertise or not.

- Feedback to crowd: This feature is only mentioned in one mixed paper, and it is not strange why the industry has no interest in giving feedback to the crowd, as industry is more concerned with the crowdsourced task and its quality.

- Knowledge sharing: This feature, which is more like an intrinsic incentive, is not interesting for industry, which is more connected with extrinsic incentives like financial ones.

- Love of community: This feature, which is another intrinsic incentive, is not interesting for industry either.

- Not known to crowdsourcer: It is interesting to find out that in industry, the crowd cannot hide from the crowdsourcer. This shows the importance the industry places on knowing the workforce, for reasons such as credibility and trustworthiness.

- Number not abundant: This crowd feature is also not mentioned in industry papers. This could mean that the industry in which crowdsourcing is being used, welcomes more and more participants, especially since crowd workforce is inexpensive in crowdsourcing platforms.

- Personal skill development: This intrinsic incentive is also not interesting for industry papers.

- Self-esteem: This intrinsic incentive is also not interesting for industry papers.

- Volunteering: Interestingly enough, this important feature has not been mentioned in any industry papers.

It is also worth mentioning that the five most notable features in academic papers were incentives provision (38 definitions), online environment (37 definitions), largeness (36 definitions), user-driven (33 definitions), and financial incentives (26 definitions). Furthermore, the five most notable features in industry papers are incentives provision (16 definitions), largeness
(13 definitions), user-driven (11 definitions), financial incentives (10 definitions), and open call (10 definitions). For mixed papers, these five are largeness (10 definitions), diversity (8 definitions), online environment (8 definitions) incentives provision (7 definitions), and user-driven ( 7 definitions). These numbers and the presence of features reflect the interests of academia and industry and what they consider most relevant to crowdsourcing. This also illustrates the three most relevant features of crowdsourcing that are present in all the above lists: incentives provision, largeness, and userdriven.

To further enrich our taxonomy of crowdsourcing, we will now discuss a set of inter-dependencies between various crowdsourcing features to help the decision of crowdsourcing developers on configuring their crowdsourcing platforms. To express dependencies, we use the popular notions of Require and Exclude in Feature Model [94]. We also use two new relations Support and Hinder, which are the lighter versions of Require and Exclude. Support means that a feature empowers or facilitates another feature. Hinder means that a feature deters or discourages another feature. The reason why these two new relations are introduced is that unlike software features where interdependencies are inflexible, crowdsourcing features are social, cognitive features and some interdependencies are qualitative. The set of the inter-relations were identified by the authors and then refined based on the feedback obtained from the anonymous reviewers in RCIS'14 conference, where it was first published. These inter-relations are as follows:

- Co-creation, a task feature, requires Collaboration, a crowd feature. By definition, co-creation needs collaboration among participants.

- Collaborative Contribution, a task feature, requires Collaboration, a crowd feature. It also requires Coordinate Crowd, a platform facility.

- Competence, a crowd feature, supports Complexity, Solvability, Problem Solving, Innovation, and Co-creation, five task features. The reason is that a competent participant will better solve complex tasks and will be more efficient in user-driven crowdsourcing activities.

- Complex for Computers, a task feature, requires Difficult to Automate, another task feature.

- Complex Tasks, a task feature, supports Divisible to Micro Tasks, another task feature. The notion 
is that complex tasks are usually broken down into smaller tasks, which are called micro tasks.

- Feedback to Crowd, a crowdsourcer feature, supports Motivation, a crowd feature. Providing feedback on the results increases the crowd's trust in the system and motivates them to participate in future tasks.

- Incentives Provision, a crowdsourcer feature, supports Largeness and Motivation, two crowd features.

- Largeness, a crowd feature, supports Diversity, a crowd feature. It hinders Coordinate Crowd, a platform facility. Coordinating a large group of participants is difficult to achieve, especially for collaborative and complex tasks.

- Not Known to Each Other, a crowd feature, excludes, Collaboration, another crowd feature. It also excludes Collaborative Contribution, a task feature. It hinders Social Incentives, a crowdsourcer feature, as the participants' identities will be hidden from each other.

- Open Call, a crowdsourcer feature, supports Diversity, Largeness, and Undefined-ness, three crowd features. It requires Provide Task Broadcast, a platform facility.

- Opt-out provision, a crowdsourcer feature, supports Largeness, Volunteering, and Motivation, three crowd features. The ability to opt-out without consequences would make the crowd more comfortable to participate.

- Privacy Provision, a crowdsourcer feature, supports Not Known to Crowdsourcer and Not Known to Each Other, two crowd features. It requires Hide Results from Others, a platform facility.

- Provide Attraction, a platform facility, supports Largeness, a crowd feature.

- Provide Authentication, a platform facility, excludes Not Known to Crowdsourcer, a crowd feature. It supports Manage Platform Misuse, another platform facility.

- Provide Ease of Use, a platform facility, supports Largeness, a crowd feature.

- Provide Feedback Loop, a platform facility, supports Feedback to Crowd, a crowdsourcer feature.
- Provide Quality Threshold, a platform facility, requires Competence, a crowd feature.

- Provide Quantity Threshold, a platform facility, requires Largeness, a crowd feature.

- Provide Skill Declaration, a platform facility, supports Provide Task Assignment, another platform facility. It hinders Not Known to Crowdsourcer, a crowd feature.

- Simple for Humans, a task feature, supports Atomic Tasks and Simple Tasks, two other task features.

- Simple Tasks, a task feature, supports Atomic Tasks and Simple for Humans, two other task features.

- Suitability, a crowd feature, hinders Undefinedness, another crowd feature. The reason is that selecting a crowd with certain suitability features hinders the idea of undefined-ness, which is choosing the crowd on a random basis and without any imposed selection procedures.

- Volunteering, a crowd feature, supports Motivation, another crowd feature. The notion is that volunteering in an activity acts as an intrinsic motivation.

\subsection{Threats to Validity}

We realize that there are limitations in our mapping study. In this subsection we will discuss these threats.

First, in our mapping study, we only considered papers in which crowdsourcing was clearly defined before being utilized throughout the paper, thus the results shown reflect that category of work. Being an inclusion criterion, this means that papers without a clear definition of crowdsourcing were not included in our mapping study. However, the relatively high number of studies we included, 113 papers out of the original 652 papers, should be sufficient to come up with a reasonable generalization. Indeed, and as a positive side of this constraint, our selection criteria let us avoid cases when the term is used without a clear meaning, which makes the study not genuinely related to crowdsourcing and closer to any participatory and collaborative approach. In our opinion, the inclusion of those papers would have led to a misleading statistics and conclusions on the topic.

Second, as it is typically the case in systematic mapping studies, we might have missed some pieces of work in the literature and we do not claim that we have found 
all the different definitions for crowdsourcing in all various fields of study. However, we say that the results drawn upon our collected sample of papers in the literature is generalizable and conclusive. The reason is that we reached a saturation level in our feature extraction so that new definitions we found after a certain point did not contain new features. On the other hand, we do not claim that the list of features for crowdsourcing is limited to our findings. As we showed earlier, new paradigms for crowdsourcing in the future may also mean new features for crowdsourcing in future definitions.

Third, we might have missed some literature which discussed crowdsourcing under different names, as the research on crowdsourcing is increasing and this could lead to some missed events. This is the case of most new concepts which might be manifested via different names. We reduced this risk by looking at related research and tracking known conferences and scholars in the area regardless of the explicit use of crowdsourcing as a first class term.

\section{Conclusion and Future Work}

In this paper we have extracted crowdsourcing features from various definitions which were found in the literature. To achieve this goal, different disciplines (computer sciences, business and management, medicine, etc.) and different publication venues (journals, conferences, workshops, etc.) were considered.

Our preliminary aim was to draw a picture of the current status of the research in the field of crowdsourcing; to help researchers and developers identify what has been done so far, to understand the features of the crowd and the crowdsourcer that are indicated in crowdsourcing definitions, and to resolve features' optionality or necessity for a crowdsourcing activity. To this end, we conducted a systematic mapping study, and we reviewed a total of 113 papers, having selected and analysed them following a protocol designed according to good practice in the empirical research field [29, 32, 33]. The feature collection phase was done after a content analysis of the definitions, while for classification purposes the authors reviewed every included paper thoroughly. Every paper studied was double checked to guarantee a vigorous study.

Our results show that there is a growing interest in the field of crowdsourcing and a rise in the number of scientific fields in which crowdsourcing has been utilized .We also noticed a lack of robust crowdsourcing definitions in both certain geographical areas (e.g., Australia) and certain scientific fields (e.g., Languages).
We believe that this work will become the ground work for future crowdsourcing studies where crowdsourcing features for the four constituents of crowdsourcing could be considered for quality assurance, motivation studies, etc. We also expect that this study will prove useful in industry, when crowdsourcing platforms are being built, studied, and evaluated. Crowdsourcing platforms could benefit greatly from this survey, since it will provide them with a good insight into the features they should implement, and the possible expectations of the crowd.

As a future work, we would like to develop a configurator tool for crowdsourcing which takes into account the causal relations between the different features of the four pillars of crowdsourcing as described in this paper. We would also develop a Wiki-like platform to allow a collaborative maintenance of our taxonomy of crowdsourcing. In other words, we would use a crowdsourcing-based approach to keep the up-to-date nature of our taxonomy and mapping study. Further research on the correlation between the features and the compatibility among them, including those related to psychological and sociological features, are also one of our future work directions.

\section{Acknowledgement}

The research was supported by an FP7 Marie Curie CIG grant (the SOCIAD Project) and by Bournemouth University through the Fusion Investment Fund (the BBB and VolaComp and BUUU projects) and the Graduate School Santander Grant for PGR Development.

\section{References}

[1] J. Howe, Crowdsourcing: A definition [Online] (June 2006). URL http://www.crowdsourcing.com/cs/2006/06/ crowdsourcing_a.html

[2] V. Chanal, M.-L. Caron-Fasan, How to invent a new busi-

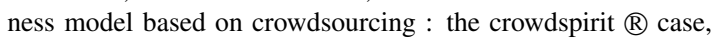
in: Conférence de l'Association Internationale de Management Stratégique, 2008, pp. 1-27.

[3] S. Djelassi, I. Decoopman, Customers' participation in product development through crowdsourcing: Issues and implications, Industrial Marketing Management 42 (5) (2013) 683-692, business Models Exploring value drivers and the role of marketing. doi:http://dx.doi.org/10.1016/j.indmarman. 2013.05.006

[4] M. K. Poetz, M. Schreier, The value of crowdsourcing: Can users really compete with professionals in generating new product ideas?, Journal of Product Innovation Management 29 (2) (2012) 245-256. doi:10.1111/j.1540-5885.2011. $00893 . \mathrm{x}$

[5] R. Ali, C. Solis, I. Omoronyia, M. Salehie, B. Nuseibeh, Social adaptation: when software gives users a voice, in: ENASE 2012: 
7th International Conference Evaluation of Novel Approaches to Software Engineering, 2012.

[6] R. Ali, C. Solis, M. Salehie, I. Omoronyia, B. Nuseibeh, W. Maalej, Social sensing: When users become monitors, in: Proceedings of the 19th ACM SIGSOFT Symposium and the 13th European Conference on Foundations of Software Engineering, ACM, New York, NY, USA, 2011, pp. 476-479. doi : 10.1145/2025113.2025196

[7] D. Pagano, B. Brügge, User involvement in software evolution practice: A case study, in: Proceedings of the 2013 International Conference on Software Engineering (ICSE), ICSE '13, IEEE Press, Piscataway, NJ, USA, 2013, pp. 953-962.

[8] D. Pagano, W. Maalej, User feedback in the appstore: An empirical study, in: Proceedings of the 2013 21st IEEE International Requirements Engineering Conference (RE), 2013, pp. 125-134. doi:10.1109/RE.2013.6636712

[9] J. M. Mortensen, M. A. Musen, N. F. Noy, Crowdsourcing the verification of relationships in biomedical ontologies, in: AMIA Annual Symposium Proceedings, Vol. 2013, American Medical Informatics Association, 2013, p. 1020.

[10] D. R. Saunders, P. J. Bex, R. L. Woods, Crowdsourcing a normative natural language dataset: A comparison of amazon mechanical turk and in-lab data collection, Journal of medical Internet research 15 (5). doi:10.2196/jmir. 2620

[11] B. Yu, M. Willis, P. Sun, J. Wang, Crowdsourcing participatory evaluation of medical pictograms using amazon mechanical turk, Journal of medical Internet research 15 (6). doi: 10.2196/jmir.2513

[12] P. Fraternali, A. Castelletti, R. Soncini-Sessa, C. V. Ruiz, A. Rizzoli, Putting humans in the loop: Social computing for water resources management, Environmental Modelling \& Software 37 (0) (2012) 68-77. doi:http://dx.doi.org/10.1016/ j.envsoft.2012.03.002

[13] H. Gao, G. Barbier, R. Goolsby, Harnessing the crowdsourcing power of social media for disaster relief, Intelligent Systems, IEEE 26 (3) (2011) 10-14. doi:10.1109/MIS. 2011.52

[14] D. MacLean, K. Yoshida, A. Edwards, L. Crossman, B. Clavijo, M. Clark, D. Swarbreck, M. Bashton, P. Chapman, M. Gijzen, M. Caccamo, A. Downie, S. Kamoun, D. Saunders, Crowdsourcing genomic analyses of ash and ash dieback - power to the people, GigaScience 2 (1) (2013) 2. doi:10.1186/ 2047-217X-2-2

[15] J. Surowiecki, The wisdom of crowds, Random House LLC, 2005.

[16] M. A. Larson, A. Said, Y. Shi, D. Tikk, A. Karatzoglou, L. Baltrunas, J. Geurts, X. Anguera, F. Hopfgarnter, Activating the crowd: Exploiting user-item reciprocity for recommendation, in: Proceedings of the first workshop on Crowdsourcing and human computation for recommender systems, ACM Conference Series on Recommender Systems, ACM RECSYS, Hong Kong, China, 2013.

[17] K. Mao, Y. Yang, M. Li, M. Harman, Pricing crowdsourcingbased software development tasks, in: Proceedings of the 2013 International Conference on Software Engineering (ICSE), ICSE '13, IEEE Press, Piscataway, NJ, USA, 2013, pp. 12051208 .

[18] J. A. Redi, T. Hoßfeld, P. Korshunov, F. Mazza, I. Povoa, C. Keimel, Crowdsourcing-based multimedia subjective evaluations: a case study on image recognizability and aesthetic appeal, in: Proceedings of the 2nd ACM international workshop on Crowdsourcing for multimedia (CrowdMM), 2013.

[19] M. J. Franklin, D. Kossmann, T. Kraska, S. Ramesh, R. Xin, Crowddb: Answering queries with crowdsourcing, in: Proceedings of the 2011 ACM SIGMOD International Conference on Management of Data, SIGMOD '11, ACM, New York, NY,
USA, 2011, pp. 61-72. doi:10.1145/1989323.1989331

[20] G. Kazai, In search of quality in crowdsourcing for search engine evaluation, in: P. Clough, C. Foley, C. Gurrin, G. Jones, W. Kraaij, H. Lee, V. Mudoch (Eds.), Advances in Information Retrieval, Vol. 6611 of Lecture Notes in Computer Science, Springer Berlin Heidelberg, 2011, pp. 165-176. doi: 10.1007/978-3-642-20161-5_17

[21] A. Foncubierta Rodríguez, H. Müller, Ground truth generation in medical imaging: A crowdsourcing-based iterative approach, in: Proceedings of the ACM Multimedia 2012 Workshop on Crowdsourcing for Multimedia (CrowdMM), CrowdMM '12, ACM, New York, NY, USA, 2012, pp. 9-14. doi:10.1145/ 2390803.2390808

[22] E. Seltzer, D. Mahmoudi, Citizen participation, open innovation, and crowdsourcing: Challenges and opportunities for planning, Journal of Planning Literature 28 (1) (2013) 318. arXiv:http://jpl.sagepub.com/content/28/1/3. full.pdf+html doi:10.1177/0885412212469112

[23] M. Bader, Crowdsourcing election monitoring in the 20112012 russian elections, East European Politics 29 (4) (2013) 521535. arXiv:http://www.tandfonline.com/doi/pdf/10. 1080/21599165.2013.818979. doi:10.1080/21599165. 2013.818979

[24] M. Bani, Crowdsourcing democracy: The case of icelandic social constitutionalism, Politics and Policy in the Information Age, Springer.

[25] C. Borch, The politics of crowds: An alternative history of sociology, Cambridge University Press, 2012.

[26] M. N. Wexler, Reconfiguring the sociology of the crowd: exploring crowdsourcing, International Journal of Sociology and Social Policy 31 (1/2) (2011) 6-20.

[27] T. Erickson, Some thoughts on a framework for crowdsourcing, in: Workshop on Crowdsourcing and Human Computation, 2011, pp. 1-4.

[28] H. Li, B. Yu, D. Zhou, Error rate analysis of labeling by crowdsourcing, in: Proceedings of the International Conference on Machine Learning 2013 Workshop: Machine Learning Meets Crowdsourcing, 2013.

[29] K. Petersen, R. Feldt, S. Mujtaba, M. Mattsson, Systematic mapping studies in software engineering, in: 12th International Conference on Evaluation and Assessment in Software Engineering, Vol. 17, 2008, p. 1.

[30] E. Engstrm, P. Runeson, Software product line testing a systematic mapping study, Information and Software Technology 53 (1) (2011) 2-13. doi:http://dx.doi.org/10.1016/j. infsof .2010.05.011

[31] A. Fernandez, E. Insfran, S. Abraho, Usability evaluation methods for the web: A systematic mapping study, Information and Software Technology 53 (8) (2011) 789-817, advances in functional size measurement and effort estimation - Extended best papers. doi:http://dx.doi.org/10.1016/j. infsof.2011.02.007

[32] J. Bailey, D. Budgen, M. Turner, B. Kitchenham, P. Brereton, S. G. Linkman, Evidence relating to object-oriented software design: A survey, in: Proceedings of the First International Symposium on Empirical Software Engineering and Measurement, IEEE Computer Society, 2007, pp. 482-484.

[33] B. Kitchenham, S. Charters, Guidelines for performing systematic literature reviews in software engineering, Tech. Rep. EBSE 2007-001, Keele University and Durham University Joint Report (2007).

[34] M. Hosseini, K. Phalp, J. Taylor, R. Ali, The four pillars of crowdsourcing: A reference model, in: Proceedings of the 2014 IEEE Eighth International Conference on Research Challenges in Information Science (RCIS), IEEE, 2014, pp. 1-12. 
[35] M. Petticrew, H. Roberts, Systematic reviews in the social sciences: A practical guide, John Wiley \& Sons, 2008.

[36] R. Wieringa, N. Maiden, N. Mead, C. Rolland, Requirements engineering paper classification and evaluation criteria: a proposal and a discussion, Requirements Engineering Journal 11 (1) (2006) 102-107. doi:10.1007/s00766-005-0021-6

[37] B. B. Bederson, A. J. Quinn, Web workers unite! addressing challenges of online laborers, in: $\mathrm{CHI}$ '11 Extended Abstracts on Human Factors in Computing Systems, CHI EA '11, ACM, New York, NY, USA, 2011, pp. 97-106. doi:10.1145/ 1979742.1979606

[38] D. Oleson, A. Sorokin, G. P. Laughlin, V. Hester, J. Le, L. Biewald, Programmatic gold: Targeted and scalable quality assurance in crowdsourcing., in: Proceedings of the Third Human Computation Workshop (HCOMP), Vol. WS-11-11 of AAAI Workshops, AAAI, 2011.

[39] O. Alonso, M. Lease, Crowdsourcing 101: Putting the wsdm of crowds to work for you, in: Proceedings of the Fourth ACM International Conference on Web Search and Data Mining (WSDM), ACM, New York, NY, USA, 2011, pp. 1-2. doi:10.1145/1935826.1935831

[40] D. DiPalantino, M. Vojnovic, Crowdsourcing and all-pay auctions, in: Proceedings of the 10th ACM Conference on Electronic Commerce, EC '09, ACM, New York, NY, USA, 2009, pp. 119-128. doi:10.1145/1566374.1566392

[41] M. Hirth, T. Hossfeld, P. Tran-Gia, Anatomy of a crowdsourcing platform - using the example of microworkers.com, in: Proceedings of the 2011 Fifth International Conference on Innovative Mobile and Internet Services in Ubiquitous Computing (IMIS), 2011, pp. 322-329. doi:10.1109/IMIS. 2011.89

[42] M. Hirth, T. Hofeld, P. Tran-Gia, Analyzing costs and accuracy of validation mechanisms for crowdsourcing platforms, Mathematical and Computer Modelling 57 (1112) (2013) 29182932, information System Security and Performance Modeling and Simulation for Future Mobile Networks. doi:http: //dx.doi.org/10.1016/j.mcm.2012.01.006

[43] Y. Singer, M. Mittal, Pricing mechanisms for crowdsourcing markets in: Proceedings of the 22nd International Conference on World Wide Web (WWW), WWW'13, International World Wide Web Conferences Steering Committee, Republic and Canton of Geneva, Switzerland, 2013, pp. 1157-1166. URL http://dl.acm.org/citation. cfm?id=2488388. 248848

[44] E. Estellés-Arolas, F. González-Ladrón-de Guevara, Towards an integrated crowdsourcing definition, Journal of Information science 38 (2) (2012) 189-200.

[45] C. Ho, S. Jabbari, J. W. Vaughan, Adaptive task assignment for crowdsourced classification, in: S. Dasgupta, D. Mcallester (Eds.), Proceedings of the 30th International Conference on Machine Learning (ICML-13), Vol. 28, JMLR Workshop and Conference Proceedings, 2013, pp. 534-542.

[46] V. M. Ribiere, F. D. D. Tuggle, Fostering innovation with $\mathrm{km}$ 2.0, Vine 40 (1) (2010) 90-101.

[47] D. A. Grier, Not for all markets, Computer 44 (5) (2011) 6-8. doi:http://doi.ieeecomputersociety.org/10. 1109/MC. 2011.155

[48] C. Heipke, Crowdsourcing geospatial data, ISPRS Journal of Photogrammetry and Remote Sensing 65 (6) (2010) 550-557, \{ISPRS\} Centenary Celebration Issue. doi:http://dx.doi. org/10.1016/j.isprsjprs.2010.06.005

[49] P. Heymann, H. Garcia-Molina, Turkalytics: Analytics for human computation, in: Proceedings of the 20th International Conference on World Wide Web (WWW), WWW'11, ACM, New York, NY, USA, 2011, pp. 477-486. doi:10.1145/ 1963405.1963473
[50] R. Kern, H. Thies, C. Zirpins, G. Satzger, Dynamic and goal-based quality management for human-based electronic services, International Journal of Cooperative Information Systems 21 (01) (2012) 3-29. arXiv:http: //www.worldscientific.com/doi/pdf/10.1142/ S0218843012400011 doi:10.1142/S0218843012400011

[51] D. L. Hansen, P. J. Schone, D. Corey, M. Reid, J. Gehring, Quality control mechanisms for crowdsourcing: Peer review, arbitration, \&\#38; expertise at familysearch indexing, in: Proceedings of the 2013 Conference on Computer Supported Cooperative Work (CSCW), CSCW '13, ACM, New York, NY, USA, 2013, pp. 649-660. doi:10.1145/2441776.2441848

[52] K. T. Stolee, S. Elbaum, Exploring the use of crowdsourcing to support empirical studies in software engineering, in: Proceedings of the 2010 ACM-IEEE International Symposium on Empirical Software Engineering and Measurement (ESEM), ESEM '10, ACM, New York, NY, USA, 2010, pp. 35:1-35:4. doi:10.1145/1852786.1852832

[53] Y. Zhao, Q. Zhu, Evaluation on crowdsourcing research: Current status and future direction, Information Systems Frontiers (2012) 1-18doi:10.1007/s10796-012-9350-4

[54] V. Ambati, S. Vogel, J. Carbonell, Towards task recommendation in micro-task markets, in: Human Computation, 2011, pp. $1-4$.

[55] T. Hossfeld, M. Hirth, P. Tran-Gia, Modeling of crowdsourcing platforms and granularity of work organization in future internet, in: Proceedings of the 23rd International Teletraffic Congress, ITC '11, International Teletraffic Congress, 2011, pp. 142-149.

[56] B. Li, D. S. Appling, S. Lee-urban, M. O. Riedl, Learning sociocultural knowledge via crowdsourced examples, in: Proceedings of the fourth AAAI Workshop on Human Computation, 2012.

[57] J. O'Neill, S. Roy, A. Grasso, D. Martin, Form digitization in bpo: From outsourcing to crowdsourcing?, in: Proceedings of the SIGCHI Conference on Human Factors in Computing Systems (CHI), CHI '13, ACM, New York, NY, USA, 2013, pp. 197-206. doi:10.1145/2470654.2470683

[58] D. E. Difallah, G. Demartini, P. Cudré-Mauroux, Mechanical cheat: Spamming schemes and adversarial techniques on crowdsourcing platforms, in: Proceedings of the First International Workshop on Crowdsourcing Web search (CrowdSearch) at 21st International World Wide Web Confernce (WWW), 2012, pp. 26-30.

[59] O. Alonso, D. E. Rose, B. Stewart, Crowdsourcing for relevance evaluation, SIGIR Forum 42 (2) (2008) 9-15. doi:10.1145/ 1480506.1480508

[60] W. Wu, W.-T. Tsai, W. Li, Creative software crowdsourcing: from components and algorithm development to project concept formations, International Journal of Creative Computing 1 (1) (2013) 57-91.

[61] Y. Baba, H. Kashima, Statistical quality estimation for general crowdsourcing tasks, in: Proceedings of the 19th ACM SIGKDD International Conference on Knowledge Discovery and Data Mining, KDD '13, ACM, New York, NY, USA, 2013, pp. 554-562. doi:10.1145/2487575.2487600

[62] J. Lehman, R. Miikkulainen, Leveraging human computation markets for interactive evolution, in: Proceedings of Machine Learning Meets Crowdsourcing Workshop at ICML 2013, 2013.

[63] A. Adepetu, A. Khaja, Y. A. Abd, A. A. Zaabi, D. Svetinovic, Crowdrequire: A requirements engineering crowdsourcing platform, in: Proceedings of 2012 AAAI Spring Symposium: Wisdom of the Crowd, 2012.

[64] R. Das, M. Vukovic, Emerging theories and models of human computation systems: A brief survey, in: Proceedings of the 2nd International Workshop on Ubiquitous Crowdsouring, 
UbiCrowd '11, ACM, New York, NY, USA, 2011, pp. 1-4. doi:10.1145/2030100.2030102

[65] M. Vukovic, Crowdsourcing for enterprises, in: 2009 World Conference on Services - I, IEEE, 2009, pp. 686-692. doi: 10.1109/SERVICES-I.2009.56

[66] A. Bozzon, M. Brambilla, S. Ceri, A. Mauri, Reactive crowdsourcing, in: Proceedings of the 22nd International Conference on World Wide Web (WWW), WWW '13, International World Wide Web Conferences Steering Committee, Republic and Canton of Geneva, Switzerland, 2013, pp. 153-164.

[67] L. Schmidt, Crowdsourcing for human subjects research, Proceedings of The World's First Conference on the Future of Distributed Work (CrowdConf).

[68] D. Brabham, Moving the crowd at istockphoto: The composition of the crowd and motivations for participation in a crowdsourcing application, First Monday 13 (6).

[69] J. Goncalves, D. Ferreira, S. Hosio, Y. Liu, J. Rogstadius, H. Kukka, V. Kostakos, Crowdsourcing on the spot: Altruistic use of public displays, feasibility, performance, and behaviours, in: Proceedings of the 2013 ACM International Joint Conference on Pervasive and Ubiquitous Computing (UbiComp), UbiComp '13, ACM, New York, NY, USA, 2013, pp. 753-762. doi:10.1145/2493432.2493481

[70] J. Pujara, B. London, L. Getoor, Reducing label cost by combining feature labels and crowdsourcing, in: ICML Workshop on Combining Learning Strategies to Reduce Label Cost, 2011.

[71] J. Ross, L. Irani, M. S. Silberman, A. Zaldivar, B. Tomlinson, Who are the crowdworkers?: Shifting demographics in mechanical turk, in: Proceedings of CHI ' 10 Extended Abstracts on Human Factors in Computing Systems, CHI EA '10, ACM, New York, NY, USA, 2010, pp. 2863-2872. doi:10.1145/ 1753846.1753873

[72] W. Mason, D. J. Watts, Financial incentives and the "performance of crowds", ACM SigKDD Explorations Newsletter 11 (2) (2010) 100-108. doi: 10.1145/1809400.1809422

[73] V. D. Mea, E. Maddalena, S. Mizzaro, Crowdsourcing to mobile users: A study of the role of platforms and tasks., in: R. Cheng, A. D. Sarma, S. Maniu, P. Senellart (Eds.), Proceedings of the First VLDB Workshop on Databases and Crowdsourcing (DBCrowd), Vol. 1025 of CEUR Workshop Proceedings, CEUR-WS.org, 2013, pp. 14-19.

[74] J. Brito, Hack, mash \& peer: Crowdsourcing government transparency, Columia Science \& Technology Law Review 9 (2007) $119-122$.

[75] E. Schenk, C. Guittard, Crowdsourcing: What can be outsourced to the crowd, and why, in: Workshop on Open Source Innovation, Strasbourg, France, 2009.

[76] G. Hsieh, Understanding and designing for cultural differences on crowdsourcing marketplaces, in: Proceedings of Chi 2011: Workshop on Crowdsourcing and Human-Computation, 2011.

[77] B. Mellebeek, F. Benavent, J. Grivolla, J. Codina, M. R. Costajussà, R. Banchs, Opinion mining of spanish customer comments with non-expert annotations on mechanical turk, in: Proceedings of the NAACL HLT 2010 Workshop on Creating Speech and Language Data with Amazon's Mechanical Turk (CSLDAMT), CSLDAMT '10, Association for Computational Linguistics, Stroudsburg, PA, USA, 2010, pp. 114-121.

[78] M. Treiber, D. Schall, S. Dustdar, C. Scherling, Tweetflows: Flexible workflows with twitter, in: Proceedings of the Third International Workshop on Principles of Engineering ServiceOriented Systems (PESOS), PESOS '11, ACM, New York, NY, USA, 2011, pp. 1-7. doi:10.1145/1985394.1985395

[79] R. Lukyanenko, J. Parsons, Conceptual modeling principles for crowdsourcing, in: Proceedings of the First International Workshop on Multimodal Crowd Sensing (CrowdSens), Crowd-
Sens '12, ACM, New York, NY, USA, 2012, pp. 3-6. doi: $10.1145 / 2390034.2390038$

[80] F. Kleemann, G. Vo, K. Rieder, Un(der)paid innovators: The commercial utiliza-tion of consumer work through crowdsourcing, Science, Technology \& Innovation Studies 4 (1).

[81] J. Howe, The rise of crowdsourcing Online (June 2006). URL http://archive.wired.com/wired/archive/14. 06/crowds.html

[82] C. Lofi, J. Selke, W.-T. Balke, Information extraction meets crowdsourcing: A promising couple, Datenbank-Spektrum 12 (2) (2012) 109-120. doi:10.1007/s13222-012-0092-8

[83] M. Porta, B. House, L. Buckley, A. Blitz, Value 2.0: Eight new rules for creating and capturing value from innovative technologies, Strategy \& Leadership 36 (4) (2008) 10-18.

[84] V. Crescenzi, P. Merialdo, D. Qiu, Wrapper generation supervised by a noisy crowd, in: R. Cheng, A. D. Sarma, S. Maniu, P. Senellart (Eds.), Proceedings of the First VLDB Workshop on Databases and Crowdsourcing (DBCrowd), Vol. 1025 of CEUR Workshop Proceedings, CEUR-WS.org, 2013, pp. 8-13.

[85] P. Whitla, Crowdsourcing and its application in marketing activities, Contemporary Management Research 5 (1).

[86] R. Cavallo, S. Jain, Winner-take-all crowdsourcing contests with stochastic production, in: B. Hartman, E. Horvitz (Eds.), Proceedings of the First AAAI Conference on Human Computation and Crowdsourcing (HCOMP), AAAI, 2013.

[87] O. Alonso, Perspectives on infrastructure for crowdsourcing, in: Workshop on Crowdsourcing for Search and Data Mining (CSDM 2011), 2011.

[88] R. Morris, Crowdsourcing workshop: the emergence of affective crowdsourcing, in: Proceedings of the 2011 annual conference extended abstracts on Human factors in computing systems, ACM, 2011.

[89] I. Abraham, O. Alonso, V. Kandylas, R. Patel, S. Shelford, A. Slivkins, H. Wu, Crowdsourcing gold-hit creation at scale: Challenges and adaptive exploration approaches, Tech. rep., Microsoft Corporation (October 2013).

[90] R. M. Araujo, 99designs: An analysis of creative competition in crowdsourced design, in: Proceedings of the First AAAI Conference on Human Computation and Crowdsourcing, 2013.

[91] D. C. Brabham, Crowdsourcing as a model for problem solving: An introduction and cases, Convergence: The International Journal of Research into New Media Technologies 14 (1) (2008) 75-90. arXiv:http://con. sagepub.com/content/14/1/ 75.full.pdf+html doi:10.1177/1354856507084420

[92] G. Adda, J. J. Mariani, L. Besacier, H. Gelas, Economic and Ethical Background of Crowdsourcing for Speech. John Wiley \& Sons, Ltd, 2013, Ch. 11, pp. 303-334. doi:10.1002/9781118541241.ch11 URL http://dx.doi.org/10.1002/9781118541241. ch11

[93] L. R. Varshney, Privacy and reliability in crowdsourcing service delivery, in: Proceedings of the 2012 Annual Service Research and Innovation Institute Global Conference (SRII), 2012, pp. 55-60. doi:10.1109/SRII.2012.17

[94] K. C. Kang, S. G. Cohen, J. A. Hess, W. E. Novak, A. S. Peterson, Feature-oriented domain analysis (foda) feasibility study, Tech. rep., Carnegie-Mellon University Software Engineering Institute (November 1990). 